\title{
Aggregation-Induced Radical of Donor-Acceptor Organic Semiconductors
}

\author{
Zhongxin Chen, ${ }^{1}$ Yuan Li $*,{ }^{1}$ Weiya Zhu, ${ }^{1}$ Zejun Wang, ${ }^{1}$ Wenqiang Li,${ }^{1}$ Miao Zeng, ${ }^{1}$ Fei \\ Huang $* 1$
}

1 Institute of Polymer Optoelectronic Materials and Devices, State Key Laboratory of Luminescent Materials and Devices, South China University of Technology, Guangzhou 510640, P. R. China

* Correspondence author: celiy@ scut.edu.cn, msfhuang@scut.edu.cn

\begin{abstract}
Narrow bandgap donor-acceptor organic semiconductors are generally considered to show closed-shell singlet ground state and their radicals are reported as impurities, polarons, charge transfer state monoradical or defects. Herein, we reported the open-shell singlet diradical electronic ground state of two diketopyrrolopyrrole-based compounds Flu-TDPP and DTPTDPP via the combination of variable temperature NMR, variable temperature electron spin spectroscopy (ESR), superconducting quantum interference device magnetometry, and theoretical calculations. It is observed that the quinoid-diradical character is significantly enhanced in aggregation state because of the limitation of intramolecular rotation. Consequently, we propose a mechanism of aggregation-induced radical to understand the driving force of the open-shell diradical formation of DTP-TDPP based on the ESR spectroscopy test in different proportions of mixed solvents. Our results demonstrate the thermally-excited triplet state for donor-acceptor organic semiconductors, providing a novel view to comprehend the intrinsic chemical structure of donor-acceptor organic semiconductors, as well as the potential electronic transition process between ground state and excited state.
\end{abstract}




\section{Introduction}

In the recent more than 30 years, organic semiconductors (OSCs) exhibited great application potential in organic light-emitting diodes (OLEDs), ${ }^{1,2}$ organic photovoltaics (OPVs), ${ }^{3,4}$ organic field-effect transistors (OFETs), ${ }^{5-7}$ organic photodetectors (OPDs) ${ }^{4}$ and other organic electronic devices $^{8}$. Most of the researchers made efforts to develop novel material systems and focused on the photo- and electron- excited states, however, the in-depth investigations of ground-state electronic structures are relatively ignored. The ground states of OSCs can be divided as the following main several types (Fig. 1). The first type is the closed-shell singlet ground state for the most extensively studied organic semiconductors. The representative examples are the triphenylamine, ${ }^{9}$ and the other relatively wide bandgap OSCs which have been widely applied in OLEDs possessing a definite singlet electronic ground state (Fig. 1a). The second type is open-shell singlet ground state $(S=0$, spin multiplicity $=1)$ for diradicals with two unpaired electrons. Over the past several decades, extensive researches on the diradical analogs including polycyclic aromatic hydrocarbons (PAHs) including zethrenes and oligothiophenes have achieved a great progress on the tuning of their ground states. ${ }^{10-16}$ For most of the diradical molecules such as para-quinodimethanes (p-QDMs) analogues (Fig. 1b), ${ }^{11}, 17$ they exhibit a singlet ground state because of the efficient delocalization of spins on $\pi$-conjugated system, giving birth to a relatively strong coupling between their two spins. The third type is the doublet ground state which existed in monoradical with one unpaired electron in single molecular structure, meaning that the spin quantum number $(\mathrm{S})$ is $1 / 2$, giving a ground-state spin multiplicity of doublet (Fig. 1c). Li et al. demonstrated the highly efficient OLEDs based on monoradical emitters with the spin doublet ground state. ${ }^{18}$ The fourth type is triplet spin states $(S=1$, spin multiplicity $=3)$ for diradicals. The previous reports on triangulene-based diradicals have also demonstrated the possibility to obtain relatively stable diradicals with triplet ground state by extending the conjugation system of phenalenyl radicals, however, this synthesis of this type of molecules are still very challenging in this field (Fig. 1d). ${ }^{10,19,20}$ 

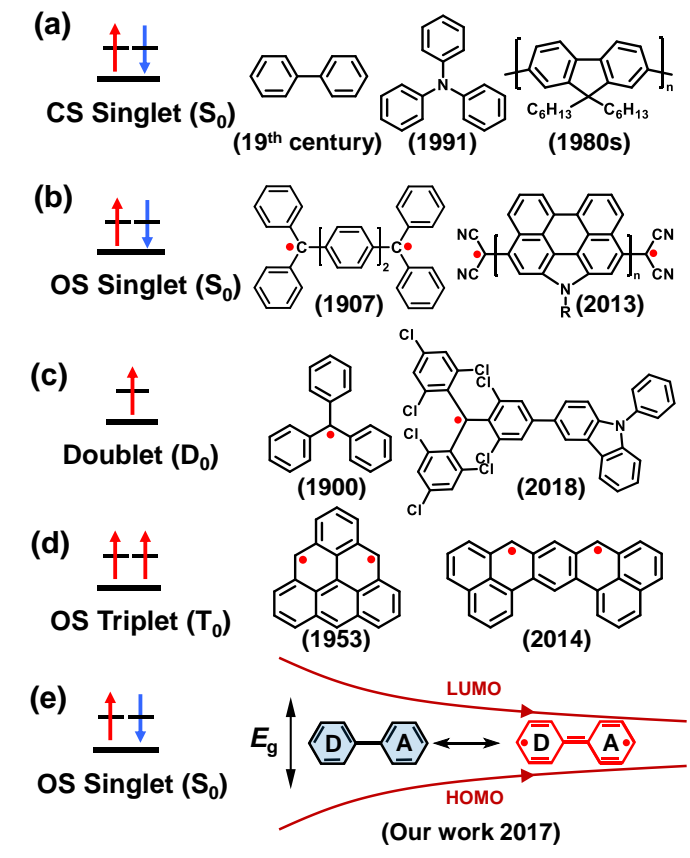

Figure 1. Typical molecules and electronic ground states of (a) closed-shell singlet molecules, (b) open-shell (OS) singlet diradicals, (c) doublet monoradicals, (d) OS triplet diradicals, and (e) donor-acceptor type narrow bandgap organic semiconductors with open-shell singlet state.

Differing from the open-shell PAH radicals and other quinoidal $p$-QDMs radicals, donoracceptor (D-A) type narrow bandgap OSCs are commonly viewed as closed-shell structure, and the radical species have been recognized as oxygen traps, ${ }^{21}$ impurities or defects, ${ }^{22,}{ }^{23}$ polarons,${ }^{24}$ or radical cation/anions ${ }^{25}$. In our previous work, we observed the intrinsic diradical character of the D-A conjugated small molecules based on various acceptor units including benzothiadiazole (BT), diketopyrrolopyrrole (DPP), and naphthalene diimide (NDI) ${ }^{26} \mathrm{We}$ proposed the quinoid-diradical resonance structure to understand the intrinsic radical ground state and the thermally-excited triplet state (Fig. 1e). ${ }^{26}$ It was worth mentioned that Prof. Bhanuprakash, Prof. Wudl and Prof. Wu proposed the open-shell diradical ground state of narrow bandgap oligomers and polymers based on benzobisthiadiazole (BBT). ${ }^{27-29}$ These reports demonstrated the open-shell diradical character of BBT-based organic semiconductor. However, our group systematically reported that all the narrow bandgap D-A OSCs show openshell singet groud state for the first time comparing previous work. ${ }^{26}$ Meanwhile, the 
mechanism of the formation of diradical ground state is not well studied and reported in previous work. ${ }^{27-29}$ To further investigate the driving force for the formation of quinoiddiradical, the underlying mechanism is still needed to be established.

In this contribution, we focused on the studies of ground-state electronic property and demonstrated the open-shell quinoid-diradical character of the diketopyrrolopyrrole (DPP)based small molecules. We proposed the mechanism for the formation of this quinoid-diradical structure as aggregation-induced radical (AIR). The detailed study and discussion were presented in the following work.

\section{Result and discussion}

From the molecular design, thienyl-diketopyrrolopyrrole (TDPP) -based derivatives were demonstrated to possess diradical character, ${ }^{26}$ and thus providing a good model to investigate the ground-state electronic structure. The donor groups dimethylfluorenyl (Flu) and dithienopyrrolyl (DTP) with different electron-donating capability result in the different degrees of intramolecular charge transfer (ICT) within these TDPP-based small molecules. Based on these considerations, the small molecules Flu-TDPP and DTP-TDPP based on D-AD structure were prepared by one-step Suzuki or Stille coupling reaction using TDPP as electron deficient chromophore (Fig. 2b) ${ }^{26}$ providing dark blue solids with gold-yellow lustra, whose chemical structure were characterized via the UV-vis-NIR absorption, ${ }^{1} \mathrm{H}-\mathrm{NMR},{ }^{13} \mathrm{C}-\mathrm{NMR}$ and MALDI-TOF-mass spectra (Fig. 3, S1-S6). The molecular configuration of $\pi$-conjugated framework and molecular orbital distribution in their relaxed ground-state geometry were predicted via density functional theory (DFT). DTP-TDPP possessed a smaller dihedral angle of $8^{\circ}$ compared with the $28^{\circ}$ of the relatively twist Flu-TDPP, indicating the more planar configuration for DTP-TDPP (Fig. 2c). These can be also demonstrated by the well-distributed highest occupied molecular orbitals (HOMOs) and lowest unoccupied molecular orbitals (LUMOs) observed for DTP-TDPP (Fig. S1). 

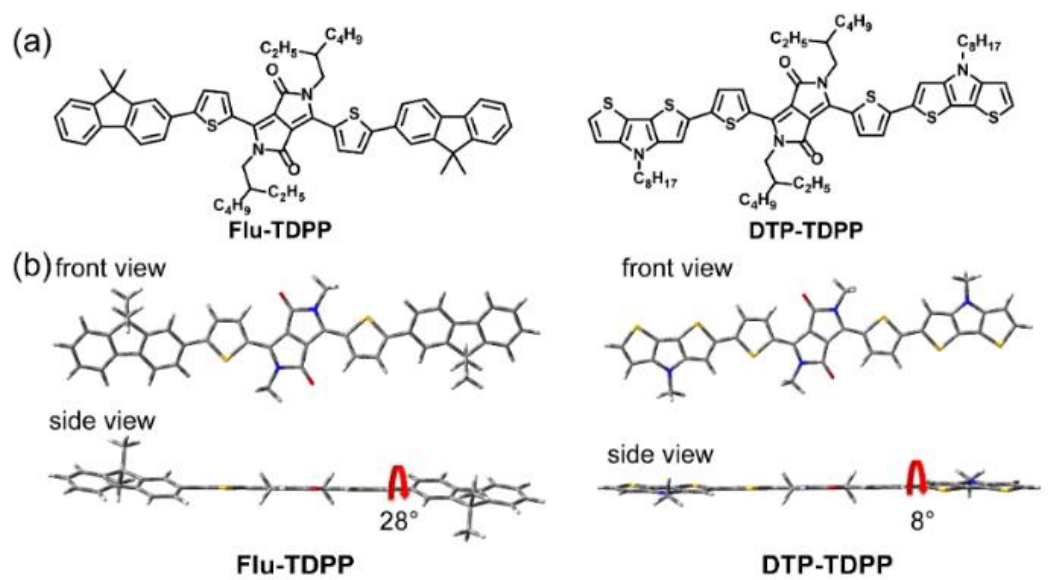

Figure 2. (a) Molecular structures of Flu-TDPP and DTP-TDPP. (b) The stimulated configurations of front views and side views of Flu-TDPP and DTP-TDPP, respectively. DTPTDPP exhibited a more planar configuration compared with Flu-TDPP.

In the film, Flu-TDPP exhibits an absorption maximum $\left(\lambda_{\max }\right)$ at $586 \mathrm{~nm}$ with an additional peak at $639 \mathrm{~nm}$, whereas DTP-TDPP shows a broad red-shifted $\lambda_{\max }$ of $640 \mathrm{~nm}$ with long absorption tail extended to over $850 \mathrm{~nm}$ (Fig. 3a). The optical bandgaps of Flu-TDPP and DTPTDPP obtained according to the absorption edge of the UV-vis-NIR spectra are 1.77 and 1.46 $\mathrm{eV}$, respectively. The smaller bandgap of DTP-TDPP implies the stronger ICT effect comparing with Flu-TDPP. The energy levels of these compounds were obtained through cyclic voltammetry measurement (Fig. S8). The HOMOs/LUMOs of Flu-TDPP and DTP-TDPP were measured to be $-5.25 /-3.48 \mathrm{eV}$ and $-4.64 /-3.18 \mathrm{eV}$, respectively. The relatively high-lying HOMO of DTP-TDPP was due to the stronger electron-donating ability of DTP group. The high-lying HOMO and narrow bandgap can promote the formation of their diradical and the thermal exciting accessibility from singlet to triplet state. ${ }^{10}$ Meanwhile, it has been widely reported that the radical character will endow the open-shell molecules with narrow HOMOLUMO bandgap. ${ }^{10,30}$ It is noteworthy to mention that there are many diradical systems based on PAHs with HOMOs higher than $-4.8 \mathrm{eV}$, however, their ESR signals are not correlated with the oxygen doping or impurity. ${ }^{31,32}$

Based on our previous work, ${ }^{24}$ electron spin resonance (ESR) was applied to study the potential radical characteristics of these two compounds. DTP-TDPP displayed a one-line ESR 
spectrum, suggesting the presence of delocalized radical (Fig. 3b). ${ }^{33}$ The $g$ value of 2.003 was in good agreement with the typical carbon-based radical rather than other defects or impurities. ${ }^{26,33,34}$ In obvious contrast, Flu-TDPP exhibited a nearly silent ESR spectrum at the same test condition. The huge differences of the ESR signal between the two compounds are related with their different molecular geometric configuration and electronic structure according to our previous work. ${ }^{26}$ DTP-TDPP exhibited a more planar quinoidal configuration due to its much narrower bandgap and intensive aggregation character comparing with FluTDPP according to the DFT calculation (Fig. 2b) and the UV-vis-NIR absorption spectra (Fig. 3a).
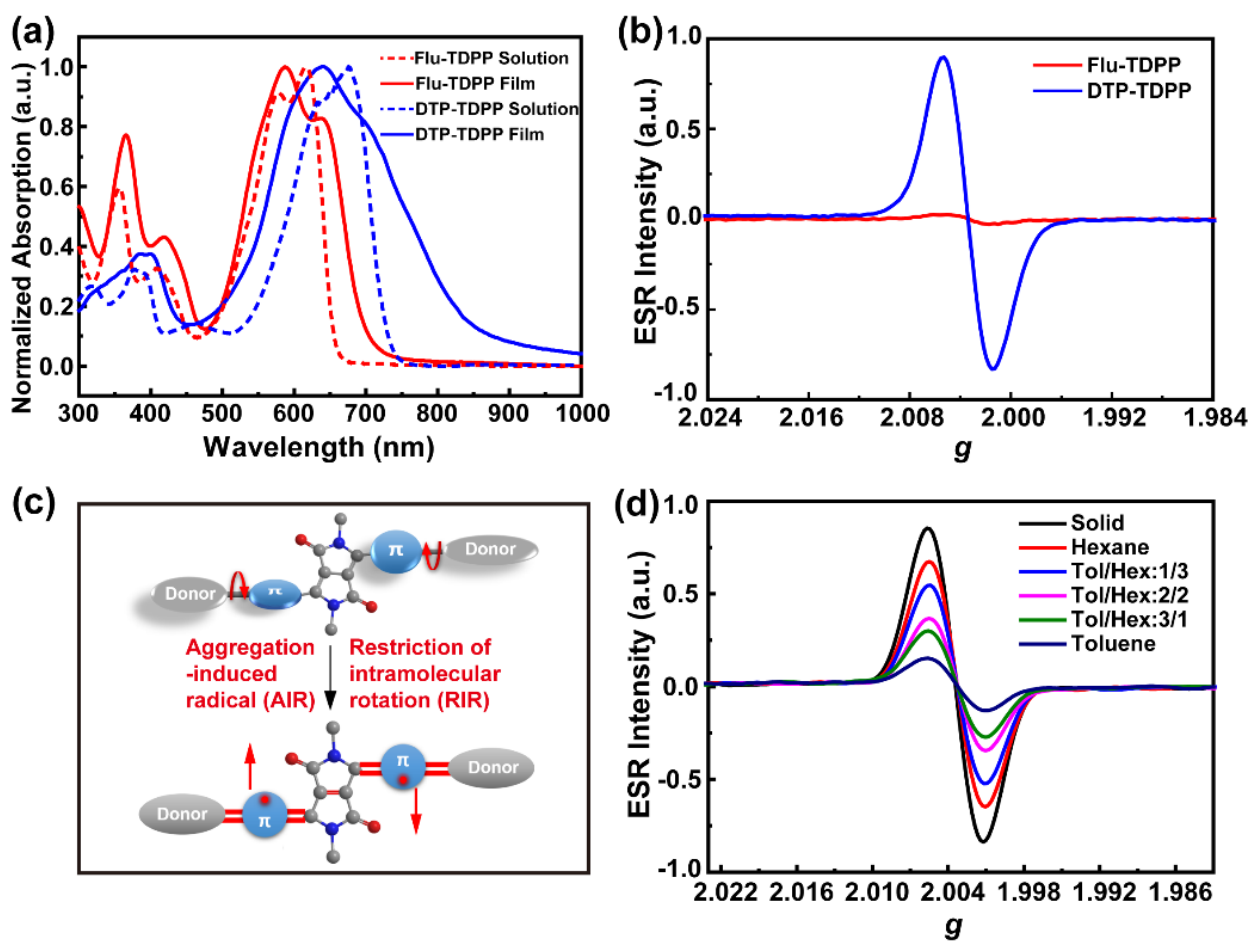

Figure 3. (a) UV-vis-NIR spectra of Flu-TDPP and DTP-TDPP in solution and film, respectively. (b) ESR spectra of Flu-TDPP and DTP-TDPP powders (both with $0.01 \mathrm{mmol}$ ). (c) The resonance structure of DPP-based molecule and the generation of radicals due to RIR and AIR effect. (d) ESR spectra of DTP-TDPP $(0.01 \mathrm{mmol})$ in different proportions of mixed solvents.

To further understand the obvious difference of the ESR signal of the two compounds, the radical character index of these two molecules was also demonstrated by the calculated diradical character, $y_{0}$ (diradical character index) and $y_{1}$ (tetraradical character index), where $y_{\mathrm{i}}$ 
$(i=0,1)$ can have values as $y_{\mathrm{i}}=0$ (closed-shell), $0<y_{\mathrm{i}}<1$ (intermediate open-shell), and $y_{\mathrm{i}}=$ 1 (pure open-shell). The $y_{0}$ of Flu-TDPP and DTP-TDPP is 0.317 and 0.382 (Table S1), respectively, indicating the weakened covalency within the bonds of these two molecules and the generation of diradicals. ${ }^{35}$

In our previous work, we proposed that the radicals in donor-acceptor type narrow bandgap organic semiconductors originated from the resonant conversion from aromatic to planar quinoids. ${ }^{26}$ Considering that the aggregation effect acts as an important role for the formation of quinoidal molecular conformations, the aggregation behavior will be fundamentally consistent with the quinoid-diradical character. The aggregation behavior of Flu-TDPP and DTP-TDPP was investigated by the variable temperature UV-vis-NIR absorption spectroscopy in solutions and thin films. In the dilute chlorobenzene solution $\left(10^{-4} \mathrm{M}\right)$ solutions of the compounds, the 0-1 and 0-0 peaks that denote the intramolecular charge transfer and aggregation characteristics, respectively, exhibited a significant reduction of the from 20 to $100{ }^{\circ} \mathrm{C}$ (Fig. S9a, S9b), which is a typical behavior for disaggregation at elevated temperature. ${ }^{36,}$ ${ }^{37}$ For Flu-TDPP in thin film, the 0-0 peak weakened and exhibited a slight red-shift (nearly 7 nm) from 20 to $100{ }^{\circ} \mathrm{C}$ (Fig. S9c). However, for DTP-TDPP, the enhanced and broad red shift (over $50 \mathrm{~nm}$ ) 1-0 peak was observed when the temperature elevated from 20 to $100{ }^{\circ} \mathrm{C}$ (Fig. S9d), demonstrating the stronger aggregation of DTP-TDPP compared with Flu-TDPP. ${ }^{38}$

To investigate the aggregation effects on the spontaneous diradical character in narrow bandgap D-A OSCs, we conducted the ESR measurements in a mixed solution with different proportions of good and poor solvents (Table S2). With the increase of poor solvent (hexane), the ESR signals increased gradually and achieved the peak in solid sample (Fig. 3d). It was due to the different aggregation behaviors in different mixed solvents. In good solvents, the DTP rings linked with TDPP core tend to rotate due to lower kinetics energy. ${ }^{39,40}$ The distorted geometric conformation will increase the solubility and weaken the aggregation of molecules in solution, thereby it is not conductive for the formation of quinoid-diradicals. On the other 
hand, when the ESR test of compounds was conducted in relatively poor solvents, strong repulsions between the solute and the solvent limited the dispersity of the molecules, which induced the formation of large-scale aggregated molecular clusters. In this way, the intramolecular rotation will be limited, leading to the more planar configuration, which theoretically enlarges the conjugated systems and stabilizes the quinoid-diradical structure (Fig. 3c). ${ }^{41,42}$ Therefore, the enhanced radical character is attributed to the enhancement of the molecular geometric planarity and we propose aggregation-induced radical (AIR) to interpret the driving force for the formation of quinoid-diradical of DTP-TDPP. The solvent effect on the ESR signal was also eliminated by experimental result and it showed the solvent effect is a negligible factor for the ESR signal decrease. Interestingly, Flu-TDPP did not exhibited an obvious tail absorption in NIR range comparing with DTP-TDPP (Fig. 3a). The long-tail absorption extended to over $800 \mathrm{~nm}$ in thin film of DTP-TDPP are indicative of the diradical contribution and the low-lying double exciton state $(\mathrm{H}, \mathrm{H}$ to $\mathrm{L}, \mathrm{L}){ }^{43}$ Meanwhile, it is noteworthy to mention that the benzobis(thiadiazole) (BBT) is typical quinoidal building block for the construction of near-infrared absorption and emission OSCs. ${ }^{41,}{ }^{42} \mathrm{We}$ also detected obvious ESR signal in the OSCs containing BBT core as well as almost all the NIR OSCs with narrow bandgap. ${ }^{24}$ We would like to highlight that the typical quinoid cores such as BBT and others will produce more quinoid-diradical configuration in aggregated state than solution state. It is well-known and reported that diradicaloids always show low photoluminescence quantum yields (PLQYs) from decay of the singlet exited state due to the absorption and transition between the $\mathrm{H}, \mathrm{H}$ and $\mathrm{L}, \mathrm{L}$ orbitals, ${ }^{43}$ singlet fission, ${ }^{44}$ and non-radiative decay pathways. ${ }^{45}$ The OSCs containing BBT core with more planar molecular structure exhibited aggregation-caused quenching (ACQ) behavior, however, the compounds with BBT core with relatively twist configuration showed aggregation-induced emission (AIE). ${ }^{41,42}$ The ACQ behavior can be well understood with our AIR mechanism and the formation of quinoid-diradical configuration will be difficult for the AIE molecules in both solution and solid states. ${ }^{26,41,42}$ 
The electronic ground states of Flu-TDPP and DTP-TDPP were investigated through variable temperature ${ }^{1} \mathrm{H}-\mathrm{NMR}$ spectra. The electronic ground states of Flu-TDPP and DTP-TDPP were investigated through variable temperature ESR and NMR spectra. Both Flu-TDPP and DTPTDPP exhibit the enhanced ESR signal with the increase of temperature (Fig. S10), implying for the diradical character with singlet ground state $\left(\mathrm{S}_{0}\right)$ as well as the thermally accessible triplet state $\left(T_{t}\right)$. The variable temperature ${ }^{1} \mathrm{H}-\mathrm{NMR}$ on Flu-TDPP gives the further evidence. The electronic ground states of Flu-TDPP and DTP-TDPP were investigated through variable temperature ${ }^{1} \mathrm{H}-\mathrm{NMR}$ spectra. For Flu-TDPP in $\mathrm{C}_{2} \mathrm{D}_{2} \mathrm{Cl}_{4}$, the sharp peaks between $7.3-8.0$ ppm in NMR spectrum broaden upon heating from 295 to $393 \mathrm{~K}$ (Fig. S11), which is a typical characteristic of the thermal population of triplet species. ${ }^{46}$ It is noteworthy that DTP-TDPP exhibits sharp peaks between $6.9-9.0 \mathrm{ppm}$ in ${ }^{1} \mathrm{H}-\mathrm{NMR}$ spectra and the peaks do not show a broadening trend as the temperature increase (Fig. S12), which is different from the observation in Flu-TDPP. It is due to the more distorted molecular conformation of DTP-TDPP comparing with Flu-TDPP in good solvent originating from the large intramolecular steric hindrance between TDPP core and DTP unit with $n$-octyl chain. The distorted geometric conformation and weakened aggregation at high temperature weakens the quinoid-diradical character. The singlet-triplet energy gap ( $\left.\Delta E_{\mathrm{ST}}\right)$ of DTP-TDPP was estimated by superconducting quantum interference device (SQUID) in the temperature range from 2 to $400 \mathrm{~K} .{ }^{46,47}$ The product of molar magnetic susceptibility and temperature $\left(\chi_{m} \cdot T\right)$ show a linear relationship with temperature $(T)$ for DTP-TDPP from 2 to $400 \mathrm{~K}$ (Fig. 4a). The plot was fitted by modified Bleaney-Bowers equation and gave the $\Delta E_{\mathrm{ST}}=-204 \mathrm{~K}(-0.406 \mathrm{kcal} / \mathrm{mol})$, suggesting the openshell singlet ground state and the low-lying thermally accessible triplet state of DTP-TDPP. ${ }^{46}$, 47

With unusual electronic structures compared with closed-shell molecules, open-shell radical molecules show unique electronic transition in photo-, electrical- and thermal-excited processes. ${ }^{18}$ For the closed-shell molecules, two singlet spins with different orientations locate 
in the highest occupied molecular orbitals. The photoexcited electrons dissipate most of the energy by means of radiative decay (Fig. 4b). For the open-shell monoradicals, the photoexcitation of monoradical molecules in their ground states (double ground state, $\mathrm{D}_{0}$ ) generates doublet excited state $\left(D_{1}\right)$. The transition from $D_{1}$ to $D_{0}$ is spin-allowed, and thus generates efficient fluorescence emission. ${ }^{18}$ We proposed some distinctive electron transitions of open-shell diradical D-A molecules. Similar to the closed-shell molecules, open-shell D-A diradicals possess two reverse spins in their ground states $\left(\mathrm{S}_{0}\right)$, however, the spin coupling of these two spins is quite weaker compared with that of closed-shell molecules. ${ }^{33}$ In the ground states, the weakly electron pairing leads to the thermally-excited triplet $\left(\mathrm{T}_{\mathrm{t}}\right)(\mathbf{F i g}$. $\mathbf{4 b})$. The singlet-triplet splitting $\left(\Delta E_{\mathrm{ST}}\right.$, energy gap between $\mathrm{S}_{0}$ and $\left.\mathrm{T}_{\mathrm{t}}\right)$ is determined by the strength of spin coupling and can be quantified according to SQUID. ${ }^{11}$ The presence of thermally-excited triplet state has been demonstrated in the PAHs and $p$-QDMs diradical molecules, ${ }^{10,46}$ but it has not been reported in D-A type OSCs. Furthermore, we propose that the formation of $T_{t}$ in narrow bandgap D-A OSCs may show underlying interaction and electronic transition with the first triplet excited state $\left(\mathrm{T}_{1}\right)$ and triplet pair $\left(\mathrm{T}_{1} \mathrm{~T}_{1}\right)^{44}$. These electronic transitions processes are closely related to singlet fission process, ${ }^{48}$ photothermal conversion, phototheranostic, ${ }^{49-51}$ as well as the triplet excitons in room-temperature phosphorescence and organic afterglow materials. ${ }^{52-54}$ The transition between electronic ground state and excitation state for open-shell D-A diradical molecules, and the influence on the structure-property-performance relationship is still undiscovered but fascinating. 

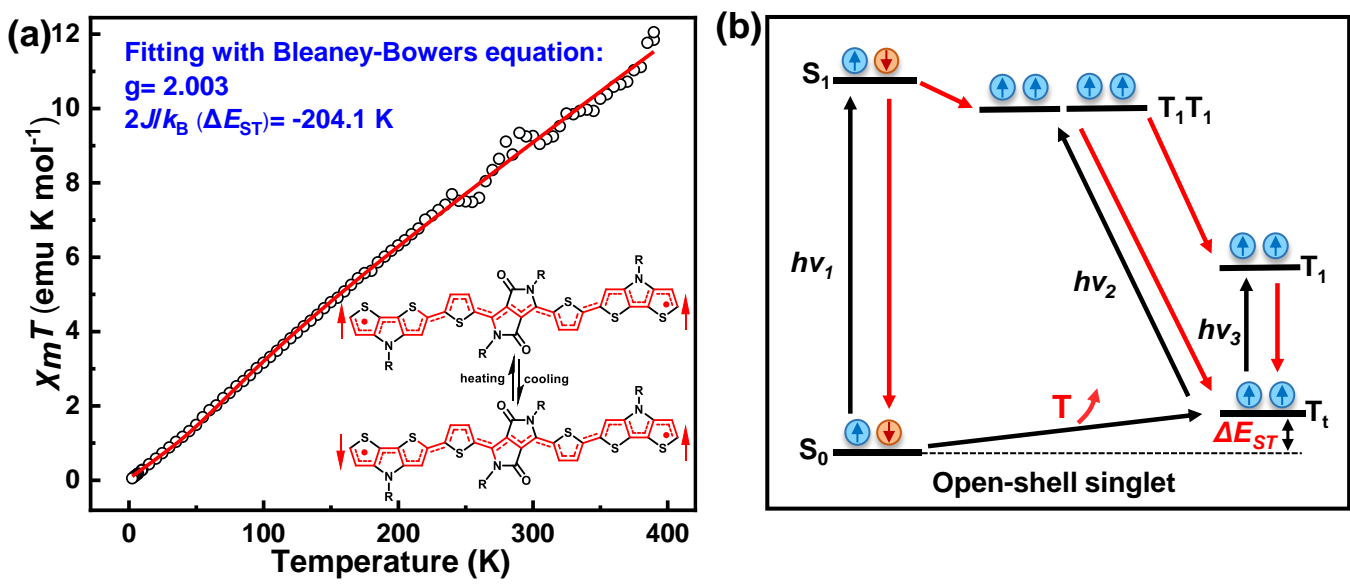

Figure 4. (a) $\chi_{\mathrm{M}} T$ vs. $T$ curve of DTP-TDPP obtained according to SQUID magnetometry. Fitting the curve to the Bleaney-Bowers equation with $g=2.003$ gives $\Delta E_{\mathrm{ST}}\left(2 J / k_{\mathrm{B}}\right)$ of -204.1 $\mathrm{K}$. Inserted figure is the resonance structures of DTP-TDPP. (b) Ground states and electronic transition of D-A type narrow bandgap molecules with open-shell singlet ground state.

\section{Conclusion}

In summary, we demonstrated the open-shell singlet ground state character of narrow bandgap D-A type OSCs originating from the generation of quinoid-diradical forms. We proposed a new concept of aggregation-induced radical to understand the enhancement of the quinoid-diradical character due to the limitation of intramolecular conformational rotation stabilizing the quinoidal diradical. The ground-state electronic structures with open-shell singlet and thermally excited triplet state provide a new viewpoint to understand the fundamental structure-property-performance relationship for narrow bandgap D-A type OSCs, as well as the design guideline for the OSCs applied in OLEDs, OPVs, OPDs, thermoelectric, photothermal, biological spintronic applications and other fields related with electron-spin states.

\section{Acknowledgements}

The authors acknowledge the financial support of the Natural Science Foundation of China (No. 51973063), Tip-top Scientific and Technical Innovative Youth Talents of Guangdong Special Support Program (2019TQ05C890), the Pearl River S\&T Nova Program of Guangzhou (201710010194), the Basic and Applied Basic Research Major Program of Guangdong 
Province (No. 2019B030302007), National Key Research and Development Program of China (No. 2019YFA0705900) funded by MOST.

\section{References}

1. Burroughes, J. H.; Bradley, D. D. C.; Brown, A. R.; Marks, R. N.; Mackay, K.;

Friend, R. H.; Burns, P. L.; Holmes, A. B., Light-emitting diodes based on conjugated polymers. Nature 1990, 347 (6293), 539-541.

2. Friend, R. H.; Gymer, R. W.; Holmes, A. B.; Burroughes, J. H.; Marks, R. N.;

Taliani, C.; Bradley, D. D. C.; Santos, D. A. D.; Brédas, J. L.; Lögdlund, M.; Salaneck, W. R., Electroluminescence in conjugated polymers. Nature 1999, 397 (6715), 121-128.

3. Hains, A. W.; Liang, Z.; Woodhouse, M. A.; Gregg, B. A., Molecular semiconductors in organic photovoltaic cells. Chem Rev 2010, 110 (11), 6689-735.

4. Peumans, P.; Yakimov, A.; Forrest, S. R., Small molecular weight organic thin-film photodetectors and solar cells. Journal of Applied Physics 2003, 93 (7), 3693-3723.

5. Meijer, E. J.; de Leeuw, D. M.; Setayesh, S.; van Veenendaal, E.; Huisman, B. H.; Blom, P. W.; Hummelen, J. C.; Scherf, U.; Kadam, J.; Klapwijk, T. M., Solution-processed ambipolar organic field-effect transistors and inverters. Nat Mater 2003, 2 (10), 678-82.

6. Sirringhaus, H.; Tessler, N.; Friend, R. H., Integrated Optoelectronic Devices Based on Conjugated Polymers. Science 1998, 280 (5370), 1741.

7. Sirringhaus, H.; Kawase, T.; Friend, R. H.; Shimoda, T.; Inbasekaran, M.; Wu, W.; Woo, E. P., High-Resolution Inkjet Printing of All-Polymer Transistor Circuits. Science 2000, 290 (5499), 2123.

8. Bijleveld, J. C.; Zoombelt, A. P.; Mathijssen, S. G. J.; Wienk, M. M.; Turbiez, M.; de Leeuw, D. M.; Janssen, R. A. J., Poly(diketopyrrolopyrrole-terthiophene) for Ambipolar Logic and Photovoltaics. Journal of the American Chemical Society 2009, 131 (46), 1661616617. 
9. Ishikawa, M.; Kawai, M.; Ohsawa, Y., Synthesis and properties of electrically conducting polytriphenylamines. Synthetic Metals 1991, 40 (2), 231-238.

10. Sun, Z.; Ye, Q.; Chi, C.; Wu, J., Low band gap polycyclic hydrocarbons: from closed-shell near infrared dyes and semiconductors to open-shell radicals. Chem Soc Rev 2012, 41 (23), 7857-89.

11. Sun, Z.; Zeng, Z.; Wu, J., Zethrenes, extended p-quinodimethanes, and periacenes with a singlet biradical ground state. Acc Chem Res 2014, 47 (8), 2582-91.

12. Takahashi, T.; Matsuoka, K.-i.; Takimiya, K.; Otsubo, T.; Aso, Y., Extensive Quinoidal Oligothiophenes with Dicyanomethylene Groups at Terminal Positions as Highly Amphoteric Redox Molecules. Journal of the American Chemical Society 2005, 127 (25), 8928-8929.

13. Ponce Ortiz, R.; Casado, J.; Hernandez, V.; Lopez Navarrete, J. T.; Viruela, P. M.; Orti, E.; Takimiya, K.; Otsubo, T., On the biradicaloid nature of long quinoidal oligothiophenes: experimental evidence guided by theoretical studies. Angew Chem Int Ed Engl 2007, 46 (47), 9057-61.

14. Casado, J.; Ponce Ortiz, R.; Lopez Navarrete, J. T., Quinoidal oligothiophenes: new properties behind an unconventional electronic structure. Chem Soc Rev 2012, 41 (17), 567286.

15. Zeng, Z.; Shi, X.; Chi, C.; Lopez Navarrete, J. T.; Casado, J.; Wu, J., Pro-aromatic and anti-aromatic pi-conjugated molecules: an irresistible wish to be diradicals. Chem Soc Rev 2015, 44 (18), 6578-96.

16. Chen, Z. X.; Li, Y.; Huang, F., Persistent and Stable Organic Radicals: Design, Synthesis, and Applications. Chem 2020.

17. Casado, J., Para-Quinodimethanes: A Unified Review of the Quinoidal-VersusAromatic Competition and its Implications. Top Curr Chem (Cham) 2017, 375 (4), 73. 
18. Ai, X.; Evans, E. W.; Dong, S.; Gillett, A. J.; Guo, H.; Chen, Y.; Hele, T. J. H.; Friend, R. H.; Li, F., Efficient radical-based light-emitting diodes with doublet emission. Nature 2018, 563 (7732), 536-540.

19. Allinson, G.; Bushby, R. J.; Paillaud, J. L.; Oduwole, D.; Sales, K., ESR spectrum of a stable triplet .pi. biradical: trioxytriangulene. Journal of the American Chemical Society 1993, 115 (5), 2062-2064.

20. Li, Y.; Huang, K.-W.; Sun, Z.; Webster, R. D.; Zeng, Z.; Zeng, W.; Chi, C.; Furukawa, K.; Wu, J., A kinetically blocked 1,14:11,12-dibenzopentacene: a persistent triplet diradical of a non-Kekulé polycyclic benzenoid hydrocarbon. Chemical Science 2014, 5 (5). 21. Schafferhans, J.; Baumann, A.; Deibel, C.; Dyakonov, V., Trap distribution and the impact of oxygen-induced traps on the charge transport in poly(3-hexylthiophene). Applied Physics Letters 2008, 93 (9).

22. Susarova, D. K.; Piven, N. P.; Akkuratov, A. V.; Frolova, L. A.; Polinskaya, M. S.; Ponomarenko, S. A.; Babenko, S. D.; Troshin, P. A., ESR spectroscopy as a powerful tool for probing the quality of conjugated polymers designed for photovoltaic applications. Chem Commun (Camb) 2015, 51 (12), 2239-41.

23. Frolova, L. A.; Piven, N. P.; Susarova, D. K.; Akkuratov, A. V.; Babenko, S. D.; Troshin, P. A., ESR spectroscopy for monitoring the photochemical and thermal degradation of conjugated polymers used as electron donor materials in organic bulk heterojunction solar cells. Chem Commun (Camb) 2015, 51 (12), 2242-4.

24. Neto, N. M. B.; Silva, M. D. R.; Araujo, P. T.; Sampaio, R. N., Photoinduced SelfAssembled Nanostructures and Permanent Polaron Formation in Regioregular Poly(3hexylthiophene). Adv Mater 2018, 30 (16), e1705052.

25. Li, S.; Yuan, N.; Fang, Y.; Chen, C.; Wang, L.; Feng, R.; Zhao, Y.; Cui, H.; Wang, X., Studies on the Bridge Dependence of Bis(triarylamine) Diradical Dications: LongRange pi-Conjugation and pi-pi Coupling Systems. J Org Chem 2018, 83 (7), 3651-3656. 
26. Li, Y.; Li, L.; Wu, Y.; Li, Y., A Review on the Origin of Synthetic Metal Radical: Singlet Open-Shell Radical Ground State? The Journal of Physical Chemistry C 2017, 121 (15), 8579-8588.

27. Thomas, A.; Bhanuprakash, K.; Prasad, K. M. M. K., Near infrared absorbing benzobis(thiadiazole) derivatives: computational studies point to biradical nature of the ground states. Journal of Physical Organic Chemistry 2011, 24 (9), 821-832.

28. Yuen, J. D.; Wang, M.; Fan, J.; Sheberla, D.; Kemei, M.; Banerji, N.; Scarongella, M.; Valouch, S.; Pho, T.; Kumar, R.; Chesnut, E. C.; Bendikov, M.; Wudl, F., Importance of unpaired electrons in organic electronics. Journal of Polymer Science Part A: Polymer Chemistry 2015, 53 (2), 287-293.

29. Tam, T. L. D.; Wu, J., Benzo[1,2-c;4,5-c' ]Bis[1,2,5]Thiadiazole in Organic Optoelectronics: A Mini-Review. Journal of Molecular and Engineering Materials 2015, 03 (01n02).

30. Zeng, Z.; Ishida, M.; Zafra, J. L.; Zhu, X.; Sung, Y. M.; Bao, N.; Webster, R. D.; Lee, B. S.; Li, R. W.; Zeng, W.; Li, Y.; Chi, C.; Lopez Navarrete, J. T.; Ding, J.; Casado, J.; Kim, D.; Wu, J., Pushing extended p-quinodimethanes to the limit: stable tetracyanooligo(N-annulated perylene)quinodimethanes with tunable ground states. J Am Chem Soc 2013, 135 (16), 6363-71.

31. Zeng, W.; Gopalakrishna, T. Y.; Phan, H.; Tanaka, T.; Herng, T. S.; Ding, J.; Osuka, A.; Wu, J., Superoctazethrene: An Open-Shell Graphene-like Molecule Possessing Large Diradical Character but Still with Reasonable Stability. J Am Chem Soc 2018, 140 (43), 14054-14058.

32. Shen, J. J.; Han, Y.; Dong, S.; Phan, H.; Herng, T. S.; Xu, T.; Ding, J.; Chi, C., A Stable [4,3]Peri-acene Diradicaloid: Synthesis, Structure, and Electronic Properties. Angew Chem Int Ed Engl 2020.

33. Abe, M., Diradicals. Chem Rev 2013, 113 (9), 7011-88. 
34. Ni, Y.; Gordillo-Gamez, F.; Pena Alvarez, M.; Nan, Z.; Li, Z.; Wu, S.; Han, Y.; Casado, J.; Wu, J., A Chichibabin's Hydrocarbon-Based Molecular Cage: The Impact of Structural Rigidity on Dynamics, Stability, and Electronic Properties. J Am Chem Soc 2020. 35. London, A. E.; Chen, H.; Sabuj, M. A.; Tropp, J.; Saghayezhian, M.; Eedugurala, N.; Zhang, B. A.; Liu, Y.; Gu, X.; Wong, B. M.; Rai, N.; Bowman, M. K.; Azoulay, J. D., A high-spin ground-state donor-acceptor conjugated polymer. Science Advances 2019, 5 (5), eaav2336.

36. Spano, F. C.; Silva, C., H- and J-aggregate behavior in polymeric semiconductors. Annu Rev Phys Chem 2014, 65, 477-500.

37. Liu, Y.; Zhao, J.; Li, Z.; Mu, C.; Ma, W.; Hu, H.; Jiang, K.; Lin, H.; Ade, H.; Yan, H., Aggregation and morphology control enables multiple cases of high-efficiency polymer solar cells. Nat Commun 2014, 5, 5293.

38. Son, S. Y.; Kim, Y.; Lee, J.; Lee, G.-Y.; Park, W.-T.; Noh, Y.-Y.; Park, C. E.; Park, T., High-Field-Effect Mobility of Low-Crystallinity Conjugated Polymers with Localized Aggregates. Journal of the American Chemical Society 2016, 138 (26), 8096-8103. 39. Liu, S.; Zhou, X.; Zhang, H.; Ou, H.; Lam, J. W. Y.; Liu, Y.; Shi, L.; Ding, D.; Tang, B. Z., Molecular Motion in Aggregates: Manipulating TICT for Boosting Photothermal Theranostics. Journal of the American Chemical Society 2019, 141 (13), 5359-5368.

40. liu, s.; li, y.; Zhang, J.; Zhang, H.; wang, y.; Chuah, C.; Tang, Y.; Lam, J.; Tsz Kin Kwok, R.; ou, h.; Ding, D.; Tang, B. Z., A Two-in-One Janus NIR-II AIEgen with Balanced Absorption and Emission for Image-Guided Precision Surgery. ChemRxiv: 2020. 41. Luo, J.; Xie, Z.; Lam, J. W.; Cheng, L.; Chen, H.; Qiu, C.; Kwok, H. S.; Zhan, X.; Liu, Y.; Zhu, D.; Tang, B. Z., Aggregation-induced emission of 1-methyl-1,2,3,4,5pentaphenylsilole. Chem Commun (Camb) 2001, (18), 1740-1.

42. Yang, J.; Fang, M.; Li, Z., Organic luminescent materials: The concentration on aggregates from aggregation-induced emission. Aggregate 2020, 1 (1), 6-18. 
43. Di Motta, S.; Negri, F.; Fazzi, D.; Castiglioni, C.; Canesi, E. V., Biradicaloid and Polyenic Character of Quinoidal Oligothiophenes Revealed by the Presence of a Low-Lying Double-Exciton State. The Journal of Physical Chemistry Letters 2010, 1 (23), 3334-3339.

44. Lukman, S.; Richter, J. M.; Yang, L.; Hu, P.; Wu, J.; Greenham, N. C.; Musser, A. J., Efficient Singlet Fission and Triplet-Pair Emission in a Family of Zethrene Diradicaloids. $J$ Am Chem Soc 2017, 139 (50), 18376-18385.

45. Jiao, Y.; Liu, K.; Wang, G.; Wang, Y.; Zhang, X., Supramolecular free radicals: near-infrared organic materials with enhanced photothermal conversion. Chem Sci 2015, 6 (7), 3975-3980.

46. Li, Y.; Heng, W. K.; Lee, B. S.; Aratani, N.; Zafra, J. L.; Bao, N.; Lee, R.; Sung, Y. M.; Sun, Z.; Huang, K. W.; Webster, R. D.; Lopez Navarrete, J. T.; Kim, D.; Osuka, A.; Casado, J.; Ding, J.; Wu, J., Kinetically blocked stable heptazethrene and octazethrene: closed-shell or open-shell in the ground state? J Am Chem Soc 2012, 134 (36), 14913-22. 47. Zhou, J.; Zhu, W.; Zeng, M.; Yang, Q.; Li, P.; Lan, L.; Peng, J.; Li, Y.; Huang, F.; Cao, Y., Aromatic inorganic acid radical. SCIENCE CHINA Chemistry 2019, 62 (16747291), 1656.

48. Rao, A.; Friend, R. H., Harnessing singlet exciton fission to break the ShockleyQueisser limit. Nature Reviews Materials 2017, 2 (11).

49. Feng, G.; Zhang, G. Q.; Ding, D., Design of superior phototheranostic agents guided by Jablonski diagrams. Chem Soc Rev 2020, 49 (22), 8179-8234.

50. Chen, C.; Ni, X.; Tian, H. W.; Liu, Q.; Guo, D. S.; Ding, D., Calixarene-Based Supramolecular AIE Dots with Highly Inhibited Nonradiative Decay and Intersystem Crossing for Ultrasensitive Fluorescence Image-Guided Cancer Surgery. Angew Chem Int Ed Engl 2020, 59 (25), 10008-10012. 
51. Chen, C.; Ni, X.; Jia, S.; Liang, Y.; Wu, X.; Kong, D.; Ding, D., Massively

Evoking Immunogenic Cell Death by Focused Mitochondrial Oxidative Stress using an AIE Luminogen with a Twisted Molecular Structure. Adv Mater 2019, 31 (52), e1904914.

52. An, Z.; Zheng, C.; Tao, Y.; Chen, R.; Shi, H.; Chen, T.; Wang, Z.; Li, H.; Deng, R.; Liu, X.; Huang, W., Stabilizing triplet excited states for ultralong organic phosphorescence. Nat Mater 2015, 14 (7), 685-90.

53. Jin, J.; Jiang, H.; Yang, Q.; Tang, L.; Tao, Y.; Li, Y.; Chen, R.; Zheng, C.; Fan, Q.; Zhang, K. Y.; Zhao, Q.; Huang, W., Thermally activated triplet exciton release for highly efficient tri-mode organic afterglow. Nat Commun 2020, 11 (1), 842.

54. Ni, X.; Zhang, X.; Duan, X.; Zheng, H. L.; Xue, X. S.; Ding, D., Near-Infrared Afterglow Luminescent Aggregation-Induced Emission Dots with Ultrahigh Tumor-to-Liver Signal Ratio for Promoted Image-Guided Cancer Surgery. Nano Lett 2019, 19 (1), 318-330. 


\section{Supporting Information}

\section{Aggregation-Induced Radical of Donor-Acceptor Organic Semiconductors}

Zhongxin Chen, ${ }^{1}$ Yuan Li $*{ }^{1}$ Weiya Zhu, ${ }^{1}$ Zejun Wang, ${ }^{1}$ Wenqiang Li, ${ }^{1}$ Miao Zeng, ${ }^{1}$ Fei

$$
\text { Huang*l }
$$

1 Institute of Polymer Optoelectronic Materials and Devices, State Key Laboratory of Luminescent Materials and Devices, South China University of Technology, Guangzhou 510640, P. R. China

* Correspondence author: celiy@ scut.edu.cn, msfhuang@scut.edu.cn 


\section{Table of contents}

Figure S1. The calculated HOMOs and LUMOs. 21

Figure S2. ${ }^{1} \mathrm{H}$ NMR spectrum of Flu-TDPP in $\mathrm{CDCl}_{3}$. 26

Figure S3. ${ }^{13} \mathrm{C}$ NMR spectrum of Flu-TDPP in $\mathrm{CDCl}_{3}$. 26

Figure S4. ${ }^{1} \mathrm{H}$ NMR spectrum of DTP-TDPP in $\mathrm{CDCl}_{3}$. 27

Figure S5. ${ }^{13} \mathrm{C}$ NMR spectrum of DTP-TDPP in $\mathrm{CDCl}_{3}$. 27

Figure S6. MALDI-TOF-MS of Flu-TDPP. 28

Figure S7. MALDI-TOF-MS of DTP-TDPP. 28

Figure S8. Cyclic voltammetry curves.

Figure S9. UV-vis-NIR spectra.

Figure S10. Variable temperature ESR spectra. 30

Figure S11. Variable temperature ${ }^{1} \mathrm{H}$ NMR spectra of Flu-TDPP. 31

Figure S12. Variable temperature ${ }^{1} \mathrm{H}$ NMR spectra of Flu-TDPP. 32 


\section{Materials}

All the reactants and catalysts used in this work were commercially available and used after high vacuum drying. Solvents used in reaction were ultra-dry (water $\leq 50 \mathrm{ppm}$ ). The crude products were purified by silica gel (300-400 mesh) column chromatography with AR eluents and recrystallization with HPLC solvent. The ${ }^{1} \mathrm{H}$ NMR spectra were measured on a Bruker AV $400 \mathrm{MHz}$ spectrometer in $\mathrm{CDCl}_{3}$ at room temperature.

\section{Synthesis}

3,6-bis(5-(9,9-dimethyl-9H-fluoren-2-yl) thiophen-2-yl)-2,5-bis(2-ethylhexyl)-2,5dihydropyrrolo[3,4-c] pyrrole-1,4-dione (Flu-TDPP)

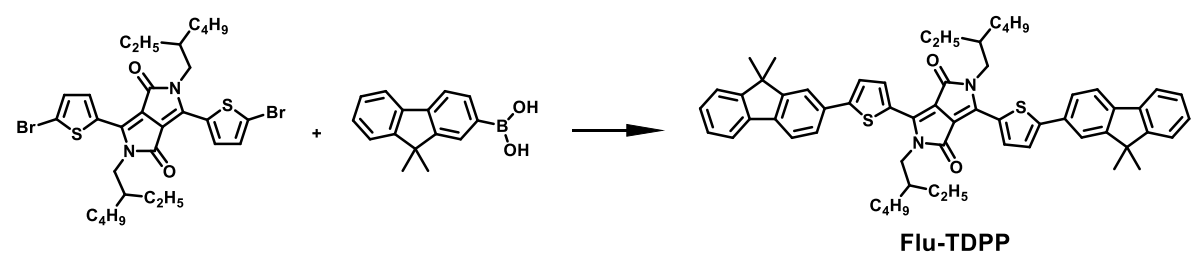

Flu-TDPP was synthesized according to previously published procedures. ${ }^{1}$ Under a nitrogen atmosphere, compound 3,6-bis(5-bromothiophen-2-yl)-2,5-bis(2-ethylhexyl)-2,5dihydropyrrolo[3,4-c] pyrrole-1,4-dione (200 mg, $0.29 \mathrm{mmol})$ was dissolved in a mixed solvent of dry toluene $(20 \mathrm{ml})$ and ethanol $(8 \mathrm{ml})$. (9,9-dimethyl-9H-fluoren-2-yl) boronic acid (174 mg, $0.73 \mathrm{mmol}), \mathrm{Na}_{2} \mathrm{CO}_{3}(3 \mathrm{ml}, 2 \mathrm{M}), \mathrm{Pd}\left(\mathrm{PPh}_{3}\right)_{4}(16.8 \mathrm{mg}, 0.0145 \mathrm{mmol})$ were added to the mixture. The resulting solution was heated to $110{ }^{\circ} \mathrm{C}$ for 24 hours. After cooling to room temperature, water was added and the aqueous layer extracted with dichloromethane. The organic layer was washed with brine, dried over anhydrous $\mathrm{MgSO}_{4}$ and then concentrated under reduced pressure. The crude product was purified by column chromatography on silica gel with a mixed solution of dichloromethane and petroleum ether (1:1) and recrystallized from mixture of ethanol and tetrahydrofuran to obtain a deep-brown solid Flu-TDPP (216 mg, 82\%). 
2,5-bis(2-ethylhexyl)-3,6-bis(5-(4-octyl-4H-dithieno[3,2-b:2',3'-d] pyrrol-2-yl) thiophen-2yl)-2,5-dihydropyrrolo[3,4-c] pyrrole-1,4-dione (DTP-TDPP)

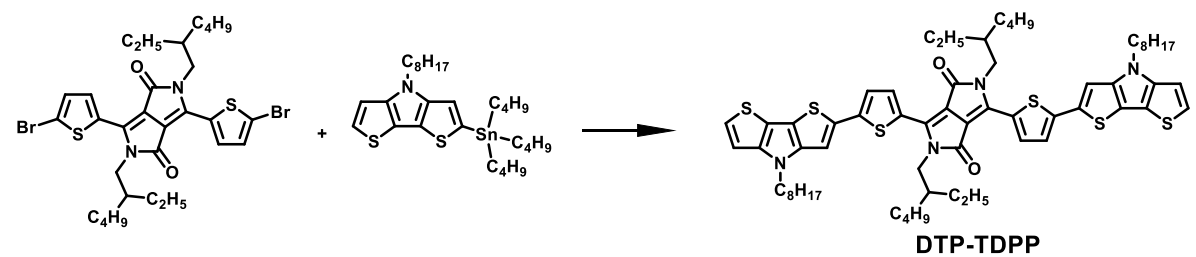

Under a nitrogen atmosphere, 4-octyl-4H-dithieno[3,2-b:2',3'-d] pyrrole (400 mg, $1.37 \mathrm{mmol}$ ) was dissolved in $20 \mathrm{ml}$ anhydrous n-hexane and cooled to $0{ }^{\circ} \mathrm{C}$. TMEDA (tetramethylethylenediamine) $(0.3 \mathrm{ml}, 1.51 \mathrm{mmol})$ was added to the solution. $n$-BuLi $(0.6 \mathrm{ml}$, $2.5 \mathrm{M}$ ) was added dropwise to the solution in 20 mins. After stirring for $2 \mathrm{~h}$ in the dark, $\mathrm{SnBu}_{3} \mathrm{Cl}$ (Tri- $n$-butyltin chloride) (491 mg $1.51 \mathrm{mmol}$ ) was added to the solution. Then returned to room temperature and stirred overnight. Water was added and the aqueous layer extracted with dichloromethane. The organic layer was washed with brine, dried over anhydrous $\mathrm{MgSO}_{4}$ and then concentrated under reduced pressure. 4-octyl-2-(tributylstannyl)-4H-dithieno[3,2- $b: 2^{\prime}, 3^{\prime}-$ d] pyrrole was obtained as dark brown liquid without further purification. Subsequently, 3,6bis(5-bromothiophen-2-yl)-2,5-bis(2-ethylhexyl)-2,5-dihydropyrrolo[3,4-c]pyrrole-1,4-dione (200 mg, $0.293 \mathrm{mmol}), 4-$ octyl-2-(tributylstannyl)-4H-dithieno[3,2- $\left.b: 2^{\prime}, 3^{\prime}-d\right]$ pyrrole (680 mg, $1.17 \mathrm{mmol})$ and $\mathrm{Pd}\left(\mathrm{PPh}_{3}\right)_{4}(16.9 \mathrm{mg}, 0.015 \mathrm{mmol})$ were dissolved in $15 \mathrm{ml} \mathrm{DMF}$ under a nitrogen atmosphere. Then the solution was heated to $120{ }^{\circ} \mathrm{C}$ and stirred for 10 hours. After cooling to room temperature, water was added and the aqueous layer extracted with dichloromethane. The organic layer was washed with brine, dried over anhydrous $\mathrm{MgSO}_{4}$ and then concentrated under reduced pressure. The crude product was purified by column chromatography on silica gel with a mixed solution of dichloromethane and petroleum ether (2:1) and recrystallized from mixture of ethanol and tetrahydrofuran to obtain dark-blue solid DTP-TDPP (194 mg, $59 \%)$.

\section{Characterizations}


Ultraviolet-visible-near infrared spectra (UV-vis-NIR) were recorded on a UV-3600 spectrophotometer. Cyclic voltammogram were carried out on CHI660e electrochemical workstation and measured in electrolyte solution of tetra-n butylammonium hexafluorophosphate $\left(\mathrm{Bu}_{4} \mathrm{NPF}_{6}\right)(0.1 \mathrm{M})$, using a $\mathrm{Hg} / \mathrm{Hg}_{2} \mathrm{Cl}_{2}$ reference electrode and a platinum counter electrode, respectively. The working electrode was glassy carbon electrode coated with the thin films of samples. HOMO energy level was determined from the onset potential of oxidation by cyclic voltammetry $E_{\mathrm{HOMO}}=-\mathrm{e}\left[E_{\mathrm{OX}}+\left(4.80-\mathrm{Fc} / \mathrm{Fc}^{+}\right)\right](\mathrm{eV}), \mathrm{LUMO}$ energy level was determined from $E_{\mathrm{LUMO}}=\left[E_{\mathrm{HOMO}}+\mathrm{e} E_{\mathrm{g}}\right.$ (optical bandgap) $](\mathrm{eV})$.

Continuous wave X-band Electron spin resonance (ESR) spectra of powder samples were performed on an A300-10/12/S-LC spectrometer. Quartz ESR standard quality tubes with an outer diameter of $4 \mathrm{~mm}$. All the powder samples were tested with operated signal attenuation of $20 \mathrm{~dB}$ at room temperature. The molar amount of test samples was $0.02 \mathrm{mmol}$. The gradient solvent experiment that investigated the aggregation dependence on radicals was conducted on solution samples with the molar quantity of solute molecules of $0.01 \mathrm{mmol}$. The solute molecules were well dissolved or dispersed in $2 \mathrm{ml}$ solvent (toluene, hexane, and their mixture). The electronic ground state was investigated by superconducting quantum interference device (SQUID) with Quantum Design 7 Tesla SQUID-VSM system. The powder samples were sealed in a plastic capsule and measured in the temperature range of 2.5 to $400 \mathrm{~K}$, with an applied field of 500 oe. The magnetic susceptibility of samples was fitted with Bleaney-Bowers equation $\left(\chi=\frac{\mathrm{N} \beta^{2} \mathrm{~g}^{2}}{3 \mathrm{kT}}\left[1+\frac{1}{3} \exp \left(\frac{J_{\mathrm{S}-\mathrm{T}}}{\mathrm{kT}}\right)\right]^{-1}\right.$, where $\chi$ is the magnetic susceptibility, $\mathrm{N}$ is Avogadro's number, $\beta$ is the Bohr magneton, $\mathrm{g}$ is the magnetic field splitting factor, $\mathrm{k}$ is the Boltzmann's constant, $\mathrm{T}$ is the temperature, and $J$ is the exchange integral) after correction of diamagnetic signal of plastic capsule and sample holder, diamagnetism of monomer and paramagnetic contamination. Density functional theory (DFT) calculations were performed to investigate the ground-state configurations using Gaussian 09 package. The Lee Yang Parr's correlation functional (B3LYP) 
that had been proved to predict the geometric structures of organic molecules and was adopted to optimize the ground state $\left(\mathrm{S}_{0}\right)$ geometry in conjunction with the $6-31 \mathrm{G}(\mathrm{d})$ basis set. The frontier molecular orbital energy levels (highest occupied molecular orbital (HOMO) and the lowest unoccupied molecular orbital (LUMO)) were calculated on the optimized structures at $\mathrm{S}_{0}$. The torsion angle between the major parts of these molecules was calculated based on the optimal ground state structure. The diradical $\left(y_{0}\right)$ and tetraradical $\left(y_{1}\right)$ characters were calculated with the spin-projected unrestricted Hartree-Fock method using Yamaguchi's formula at $\mathrm{UHF} / 6-31 \mathrm{G}(d, p)$ level of theory and basis set:

$$
y_{i}=1-\frac{2 T_{i}}{1+T_{i}^{2}} ; \quad T_{i}=\frac{n_{H O N O-i}-n_{L U N O+i}}{2}
$$

where, $i=0,1 ; T_{i}$ is the overlap between the two corresponding orbitals; $n_{\mathrm{HONO}}$ and $n_{\mathrm{LUNO}}$ are the occupation numbers of the highest occupied natural orbital and the lowest unoccupied natural orbital, respectively.

Table S1. Optical, electronic properties and diradical indexes of the materials.

\begin{tabular}{lcccccc}
\hline Materials & $\lambda_{\text {onset }^{[\mathrm{a}]}}$ & $E_{g}{ }^{\mathrm{opt}[\mathrm{b}]}$ & $\mathrm{HOMO}^{\mathrm{c}}$ & LUMO $^{\mathrm{c}}$ & $y_{0}{ }^{\mathrm{d}}$ & $y_{1}{ }^{\mathrm{e}}$ \\
& {$[\mathrm{nm}]$} & {$[\mathrm{eV}]$} & & & & \\
\hline Flu-TDPP & 699 & 1.77 & -5.25 & -3.48 & 0.317 & 0.073 \\
DTP-TDPP & 849 & 1.46 & -4.64 & -3.18 & 0.382 & 0.104
\end{tabular}

${ }^{a}$ Obtained from the absorption edge in thin films. ${ }^{b} E_{\mathrm{g}}{ }^{\mathrm{opt}}=1240 / \lambda_{\text {inter, }}{ }^{c} \mathrm{HOMO}$ was calculated according to cyclic voltammetry result, $\mathrm{E}_{\mathrm{LUMO}}=\mathrm{E}_{\mathrm{HOMO}}+E_{g}{ }^{\mathrm{opt}} \cdot{ }^{d}$ Diradical character index $\left(y_{0}\right)$ and ${ }^{e}$ tetraradical character index $\left(y_{l}\right)$ calculated with PUHF/6-31G $(d)$. All energies are in $\mathrm{eV}$, $y_{0}$ and $y_{1}$ are unitless quantities. 
Table S2. The detailed information of solvents

\begin{tabular}{|c|c|c|c|c|c|}
\hline Experiment & $\begin{array}{l}\text { Molar Amount } \\
\text { of Solute / mmol }\end{array}$ & $\begin{array}{l}\text { Radio of } \\
\text { Solvents } \\
\text { (Toluene / } \\
\text { Hexane) }\end{array}$ & $\begin{array}{l}\text { Volume of } \\
\text { Toluene / } \mu \mathrm{L}\end{array}$ & $\begin{array}{l}\text { Volume of } \\
\text { Hexane / } \mu \mathrm{L}\end{array}$ & $\begin{array}{l}\text { Total } \\
\text { Volume } \\
/ \mu \mathrm{L}\end{array}$ \\
\hline 1 & 0.01 & I & 200 & 0 & 200 \\
\hline 2 & 0.01 & $3: 1$ & 150 & 50 & 200 \\
\hline 3 & 0.01 & $1: 1$ & 100 & 100 & 200 \\
\hline 4 & 0.01 & $1: 3$ & 50 & 150 & 200 \\
\hline 5 & 0.01 & I & 0 & 200 & 200 \\
\hline
\end{tabular}
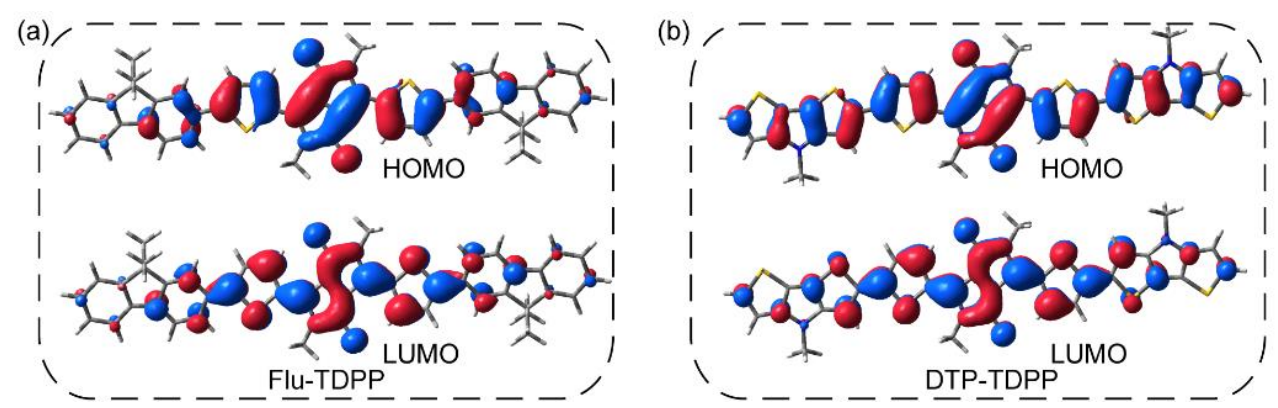

Figure S1. The calculated HOMOs and LUMOs of (a) Flu-TDPP and (b) DTP-TDPP. The latter molecule owned a more even electronic distribution. 


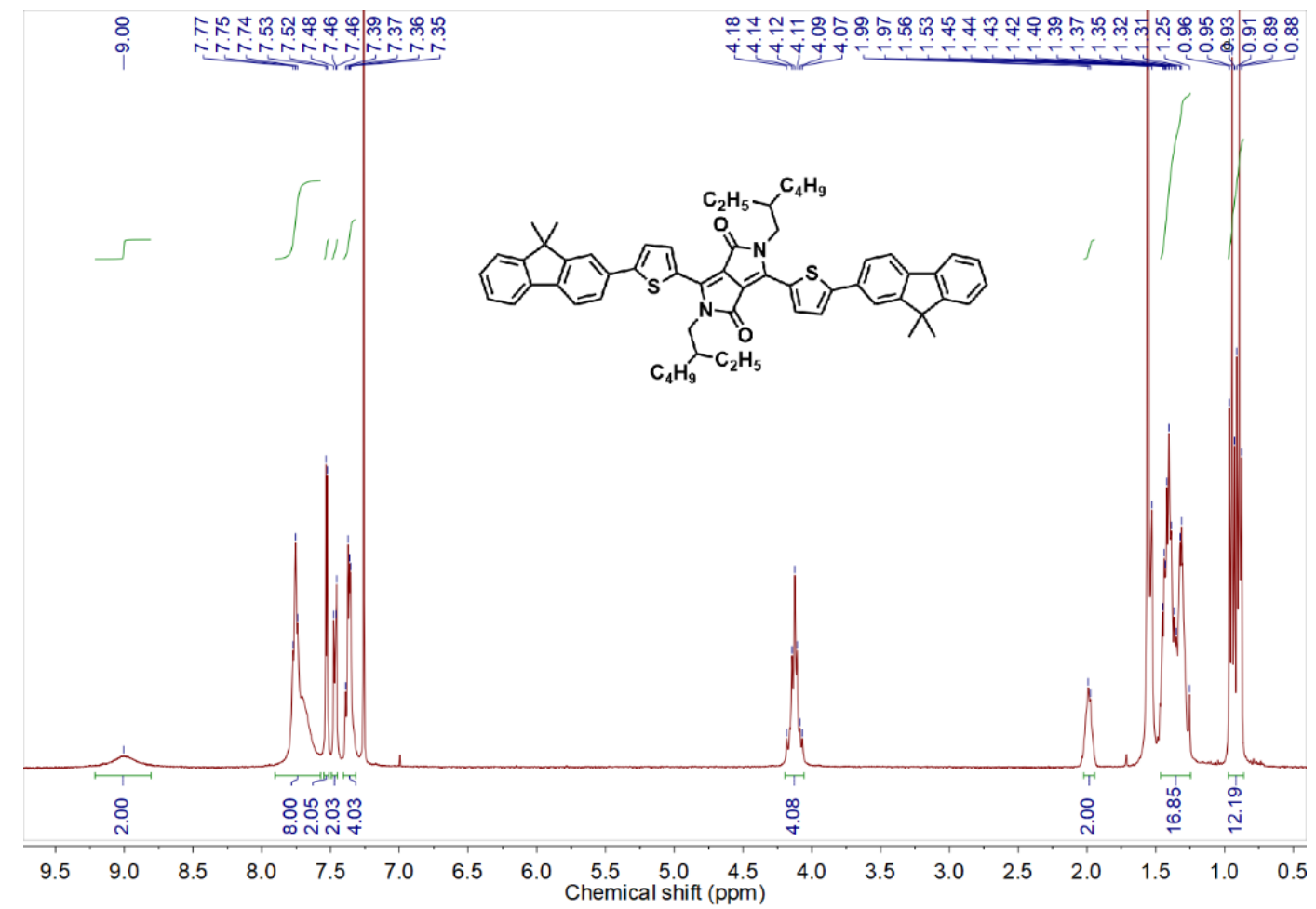

Figure S2. ${ }^{1} \mathrm{H}$ NMR spectrum of Flu-TDPP in $\mathrm{CDCl}_{3}$.

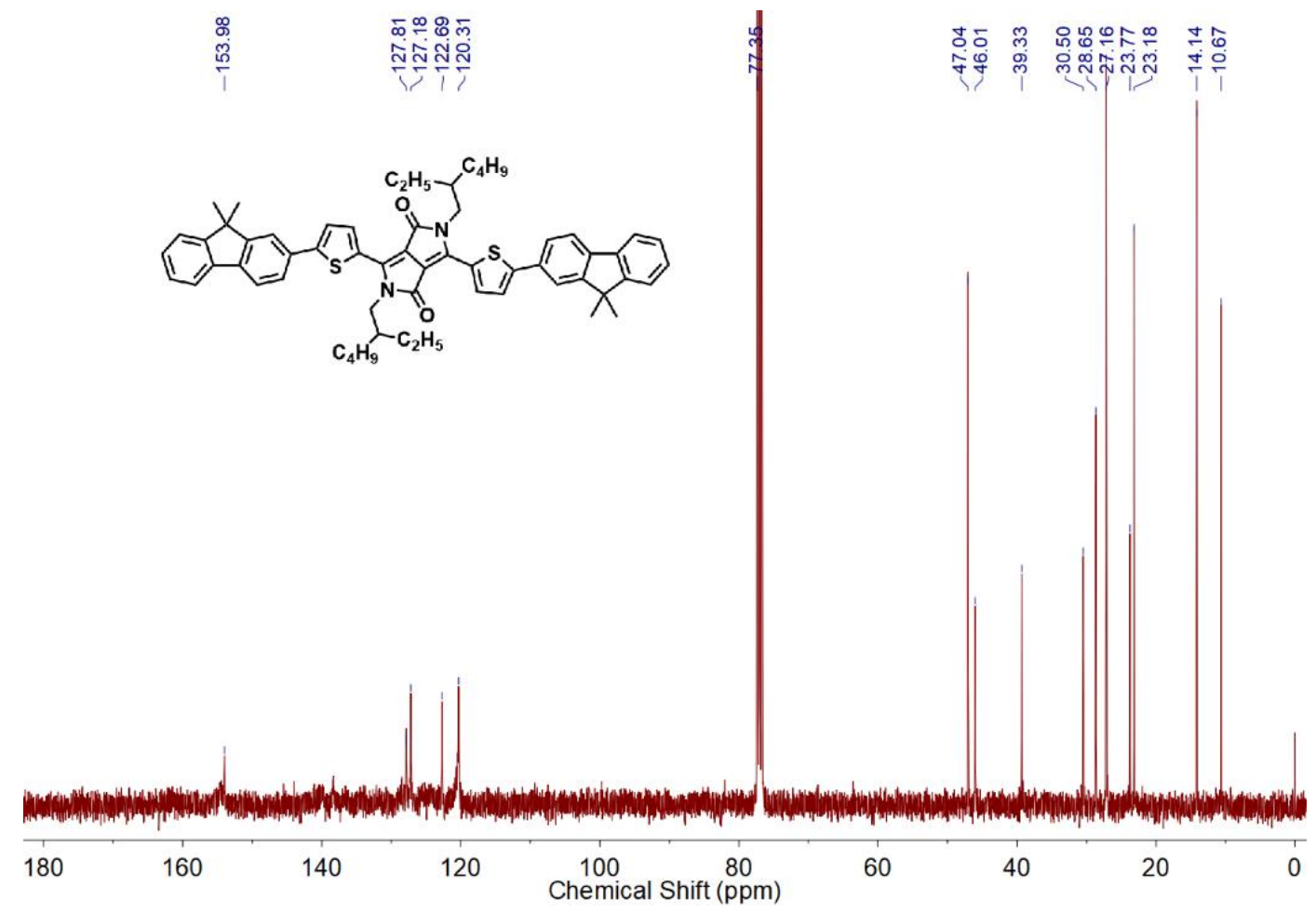

Figure S3. ${ }^{13} \mathrm{C}$ NMR spectrum of Flu-TDPP in $\mathrm{CDCl}_{3}$. 


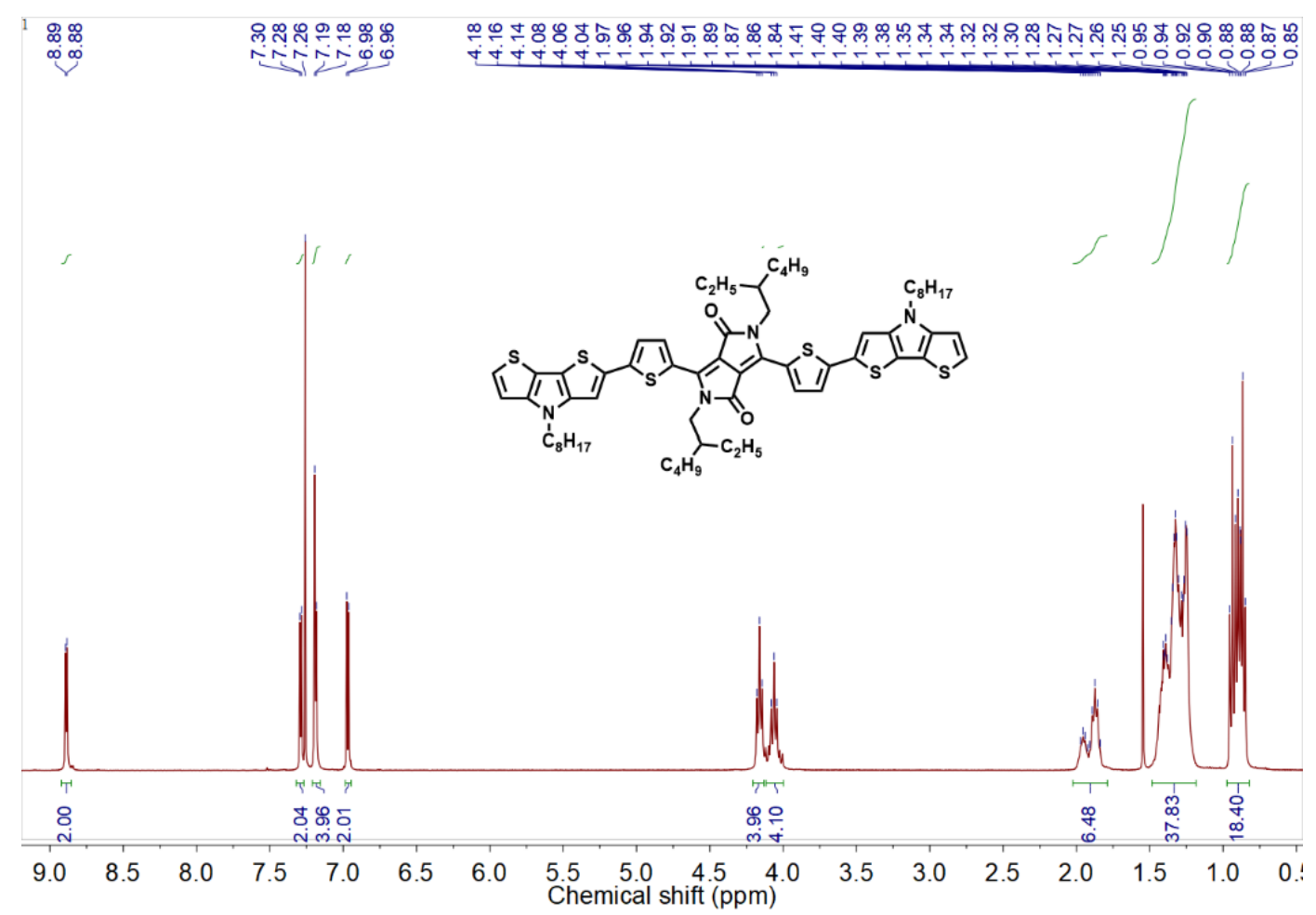

Figure S4. ${ }^{1} \mathrm{H}$ NMR spectrum of DTP-TDPP in $\mathrm{CDCl}_{3}$.
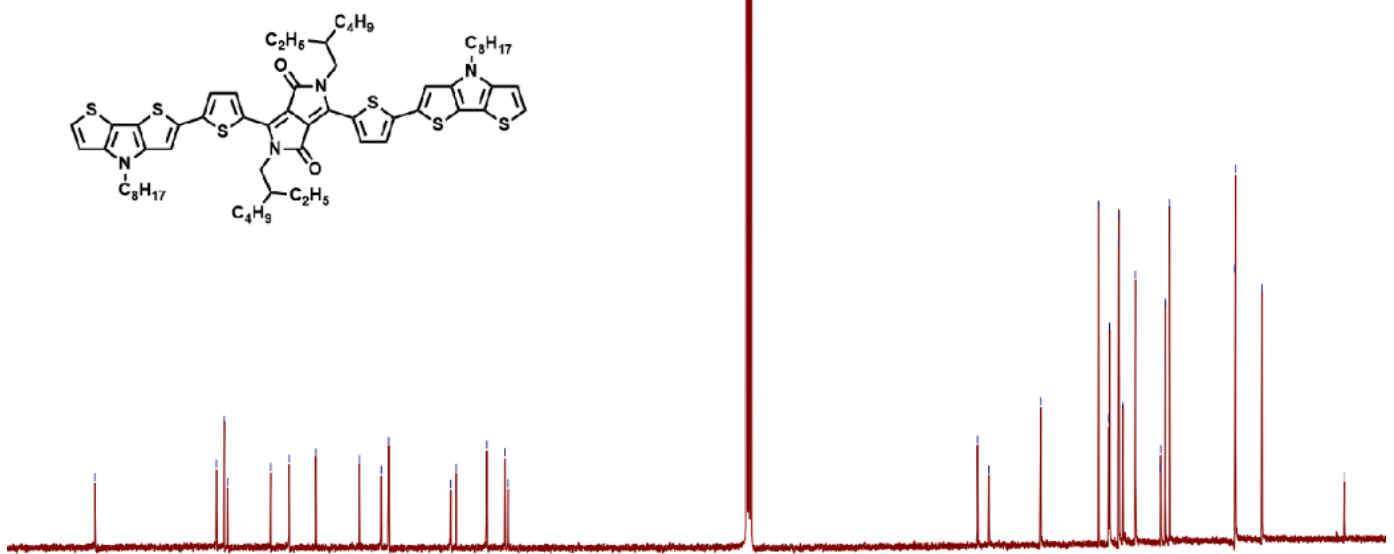

160 140 120 Chemical Shift (ppm)

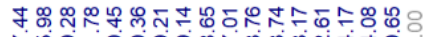

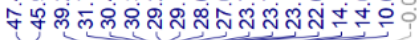

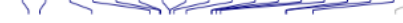

Figure S5. ${ }^{13} \mathrm{C}$ NMR spectrum of DTP-TDPP in $\mathrm{CDCl}_{3}$. 


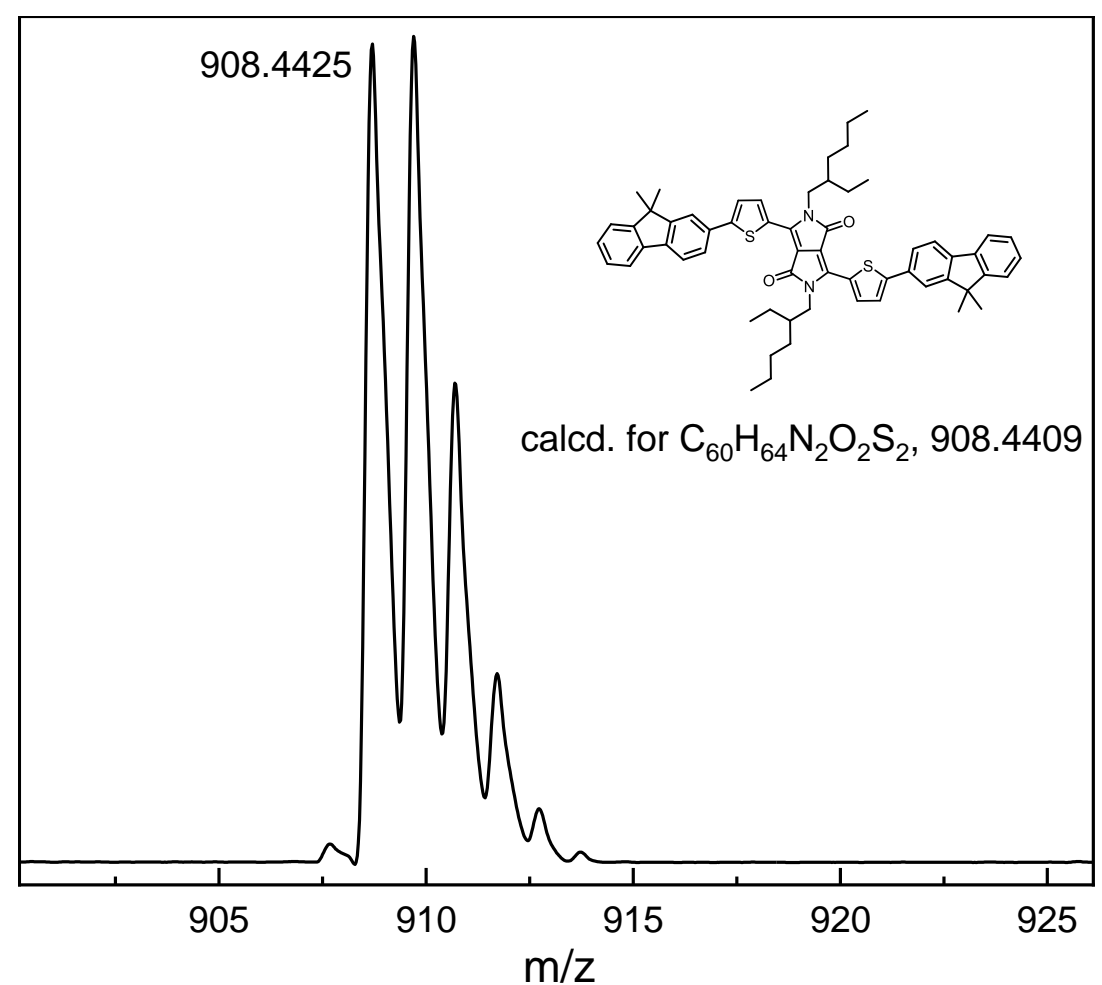

Figure S6. MALDI-TOF-MS of Flu-TDPP. Calcd for $\mathrm{C}_{60} \mathrm{H}_{64} \mathrm{~N}_{2} \mathrm{O}_{2} \mathrm{~S}_{2}: m / z: 908.4409$. Found: 908.4425 .

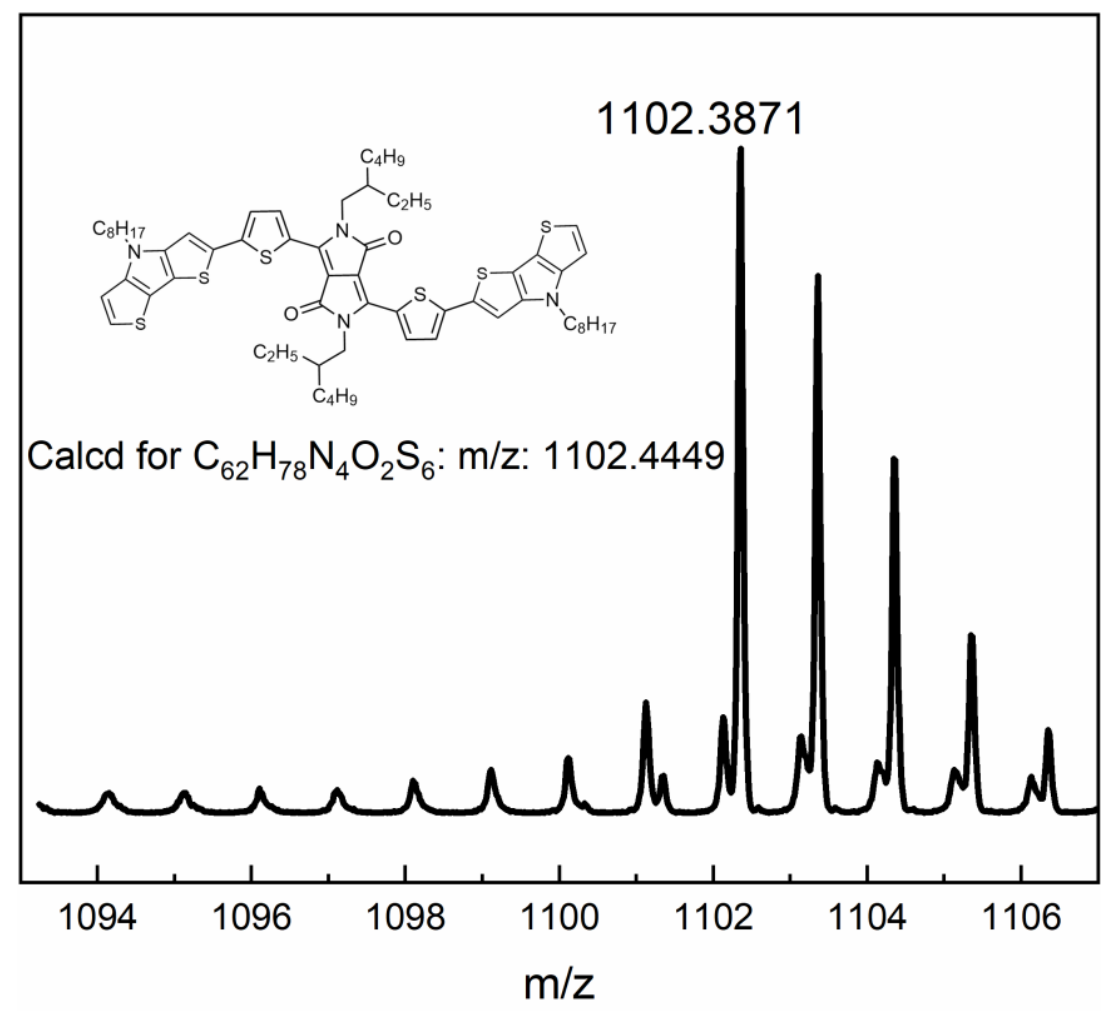

Figure S7. MALDI-TOF-MS of DTP-TDPP. Calcd for $\mathrm{C}_{62} \mathrm{H}_{78} \mathrm{~N}_{4} \mathrm{O}_{2} \mathrm{~S}_{6}: m / z: 1102.4449$. Found:1102.3871. 


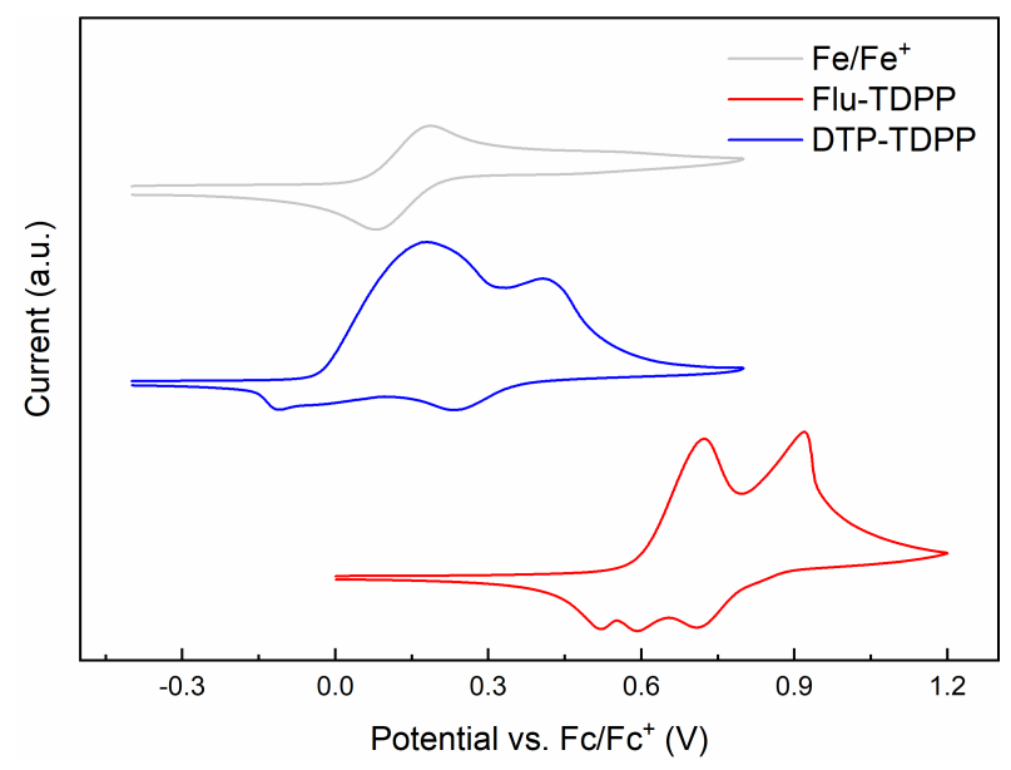

Figure S8. Cyclic voltammetry curves of Flu-TDPP and DTP-TDPP in thin films using $n$ $\mathrm{Bu}_{4} \mathrm{NPF}_{6}$ as electrolytes.
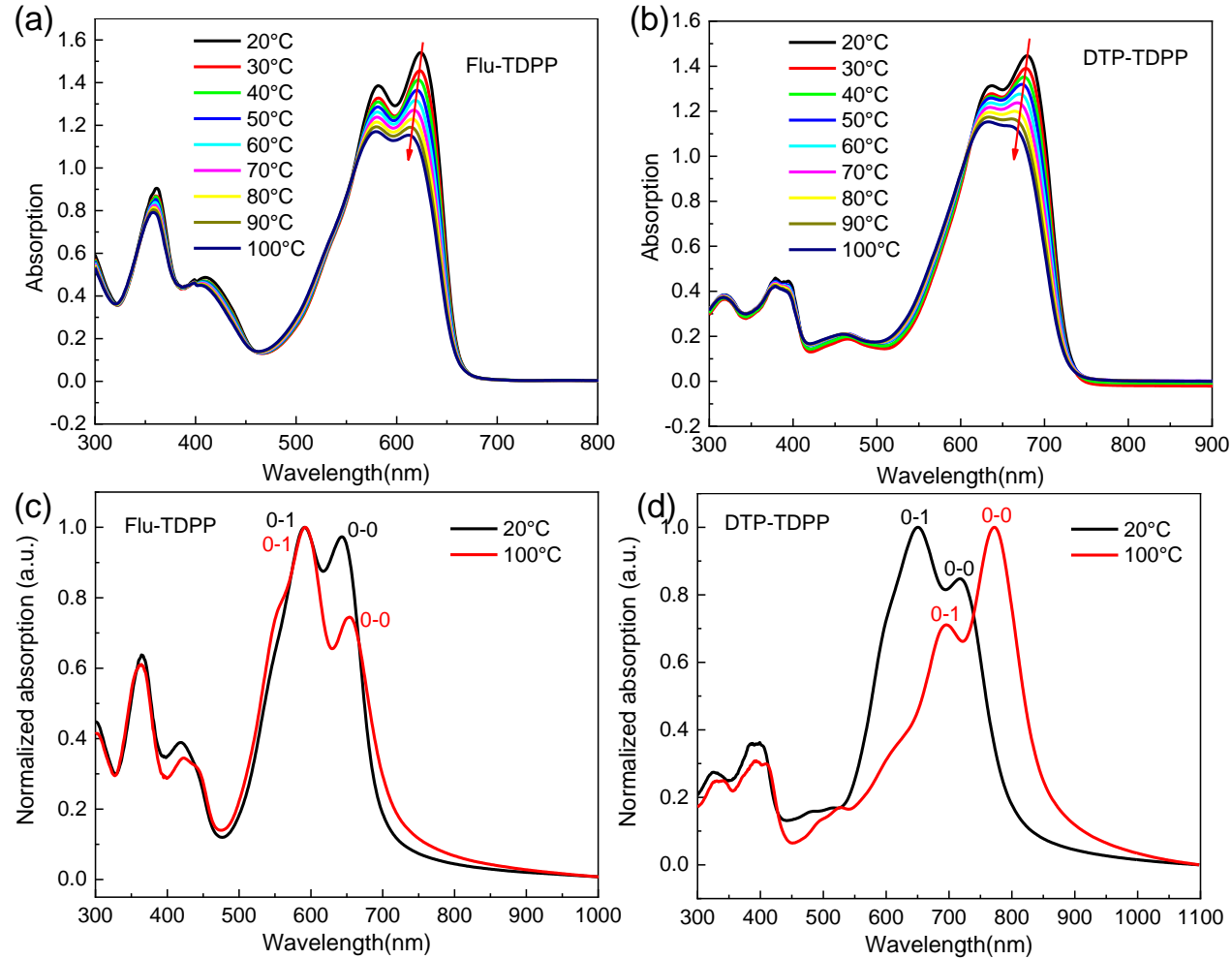

Figure S9. (a, b) UV-vis-NIR spectra of Flu-TDPP and DTP-TDPP in chlorobenzene $\left(10^{-4} \mathrm{M}\right)$ in the temperature range from $20^{\circ} \mathrm{C}$ to $100^{\circ} \mathrm{C}$, respectively. (c, d) UV-vis-NIR spectra of FluTDPP and DTP-TDPP in thin film from $20^{\circ} \mathrm{C}$ to $100^{\circ} \mathrm{C}$, respectively. 

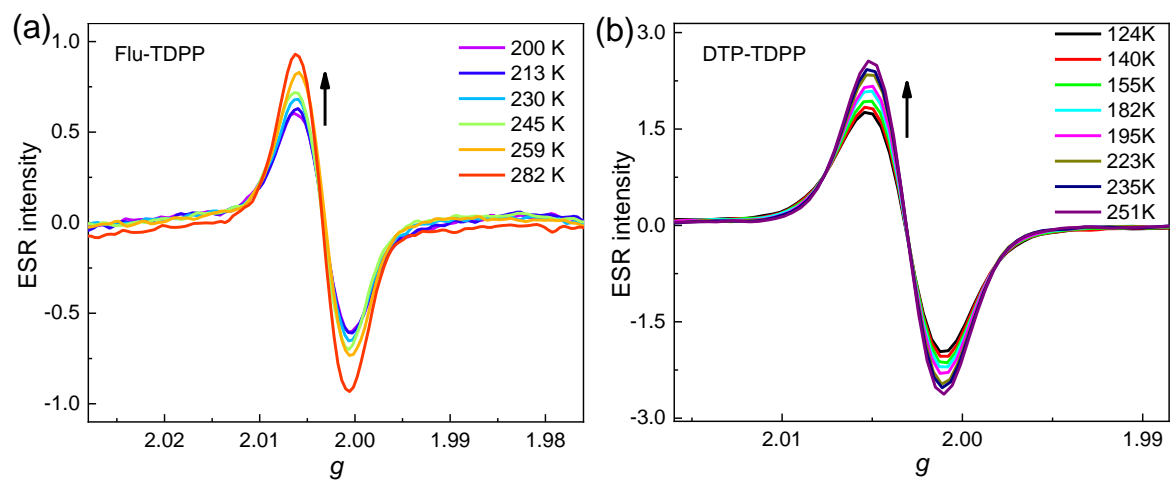

Figure S10. (a, b) Variable temperature ESR spectra of Flu-TDPP and DTP-TDPP, respectively. 

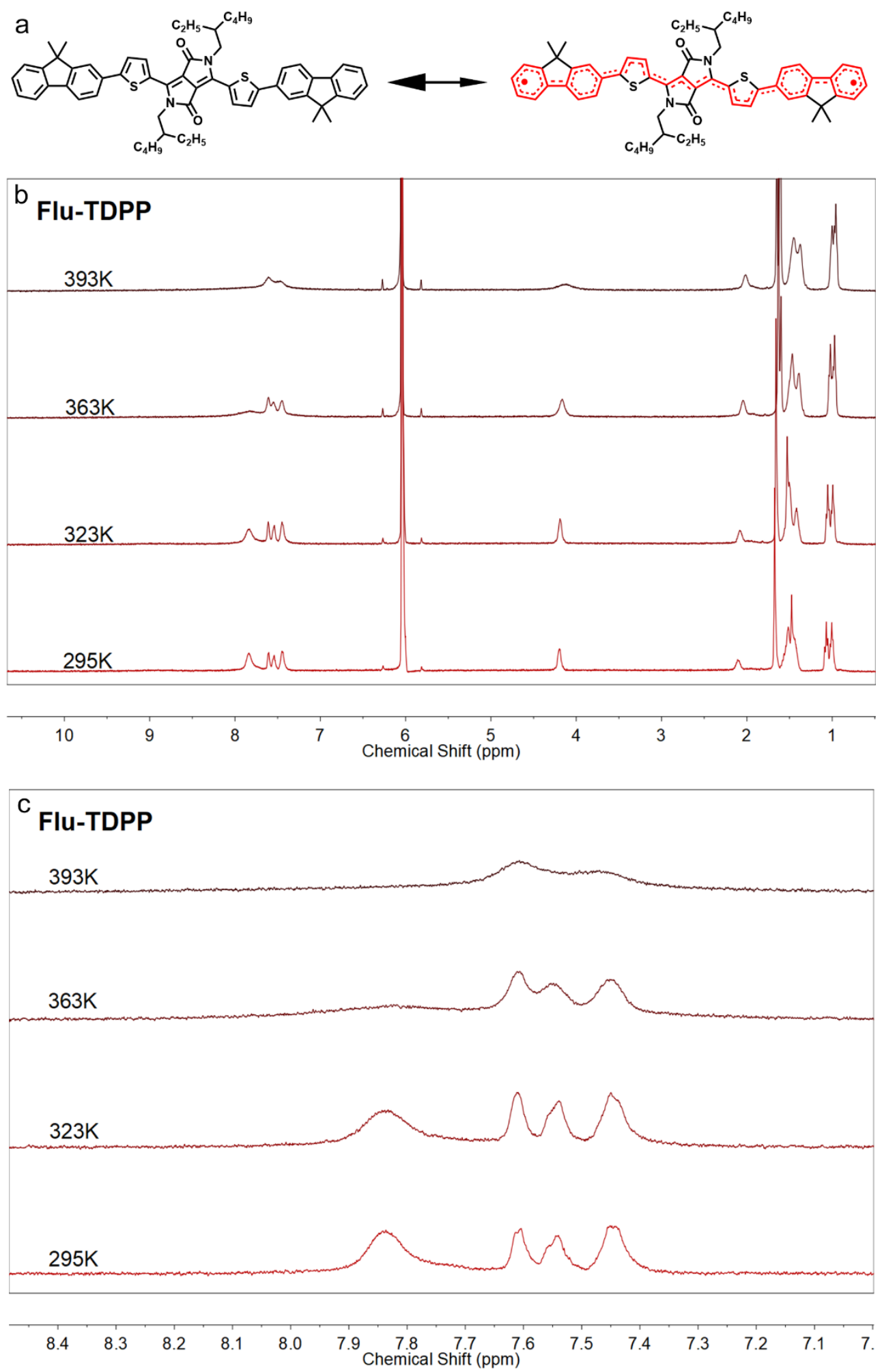

Figure S11. (a) Resonance structures of Flu-TDPP. (b) Variable temperature ${ }^{1} \mathrm{H}$ NMR spectra and (c) the enlarged spectra of Flu-TDPP in the aromatic region (7.0 to $8.6 \mathrm{ppm}$ ) in $\mathrm{C}_{2} \mathrm{D}_{4} \mathrm{Cl}_{4}$ in the temperature range from 295 to $393 \mathrm{~K}$. 
a
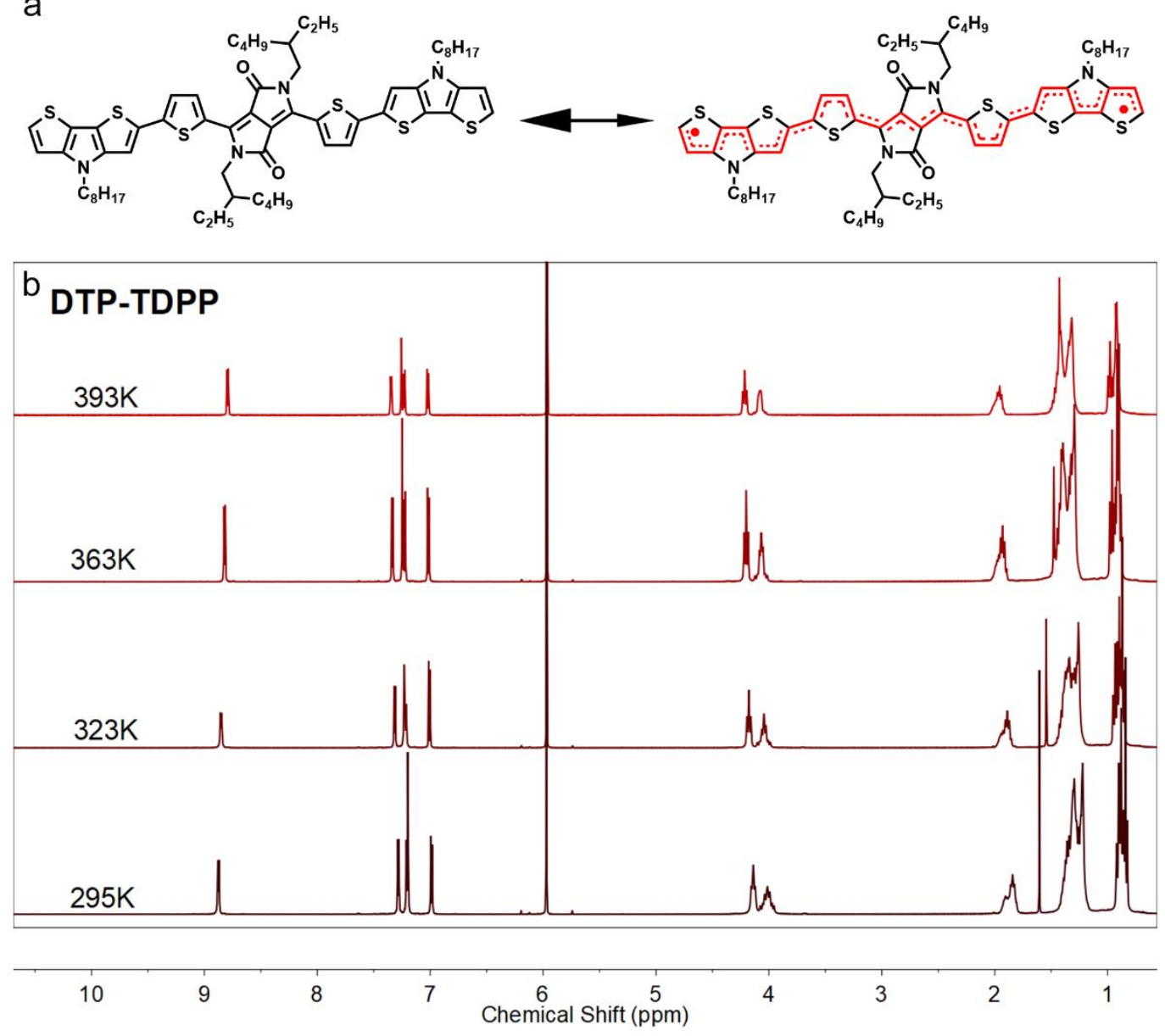

${ }^{C}$ DTP-TDPP

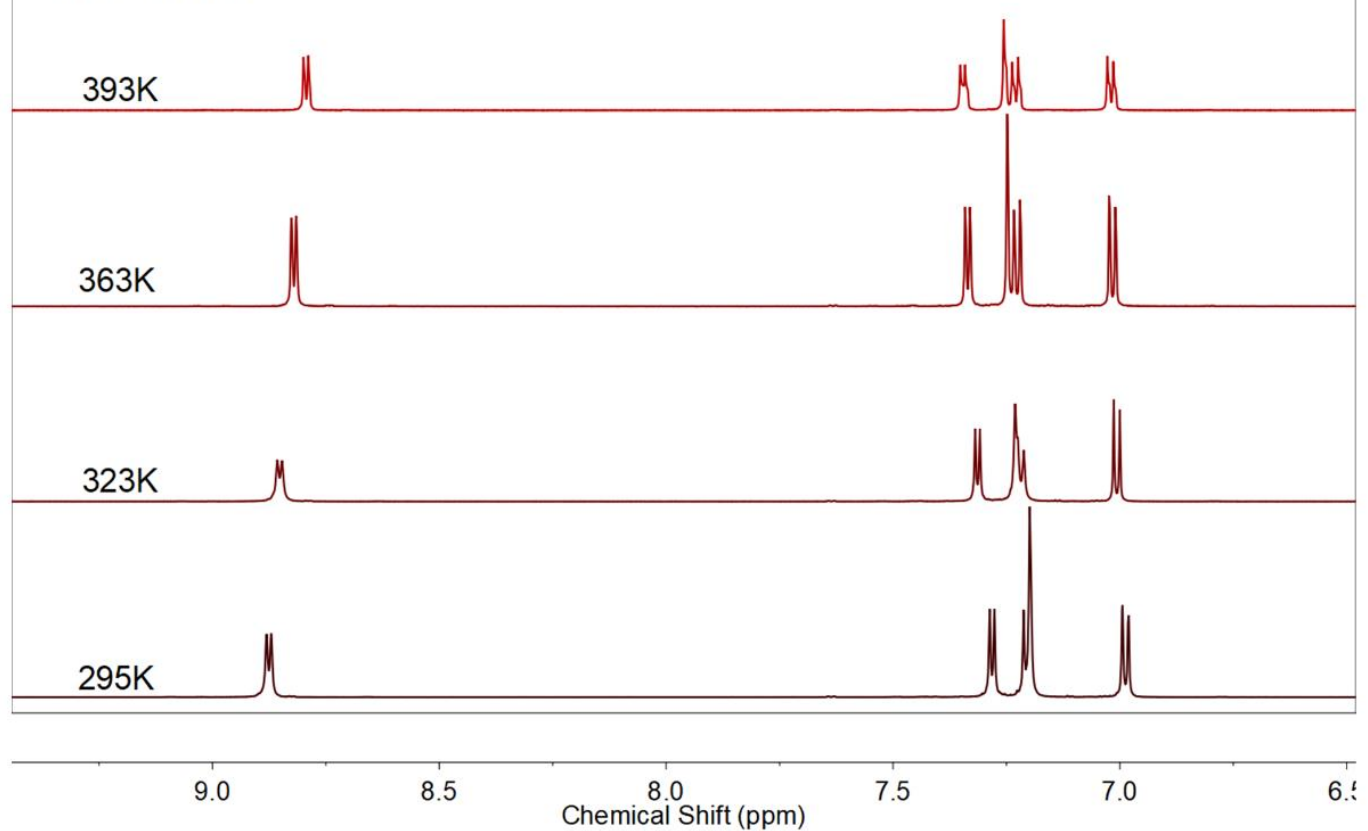

Figure S12. (a) Resonance structures of DTP-TDPP. (b) Variable temperature ${ }^{1} H$ NMR spectra and (c) the enlarged spectra of DTP-TDPP in the aromatic region (6.5 to $9.7 \mathrm{ppm}$ ) in $\mathrm{C}_{2} \mathrm{D}_{4} \mathrm{Cl}_{4}$ in the temperature range from 295 to $393 \mathrm{~K}$. 


\section{References}

1. Pang, S.; Mas-Montoya, M.; Xiao, M.; Duan, C.; Wang, Z.; Liu, X.; Janssen, R. A. J.; Yu, G.; Huang, F.; Cao, Y., Adjusting Aggregation Modes and Photophysical and Photovoltaic Properties of Diketopyrrolopyrrole-Based Small Molecules by Introducing $\mathrm{B}<--\mathrm{N}$ Bonds. Chemistry 2019, 25 (2), 564-572. 